\title{
A low computational complexity V-BLAST/STBC detection mechanism in MIMO system
}

\author{
Jin Hui Chong ${ }^{1}$, Chee Kyun $\mathrm{Ng}^{2,3 *}$, Nor Kamariah Noordin ${ }^{3}$ and Borhanuddin Mohd $\mathrm{Ali}^{3}$
}

\author{
* Correspondence: \\ mpnck@upm.edu.my \\ ${ }^{2}$ Institute of Gerontology, University \\ Putra Malaysia, UPM Serdang 43400 \\ Selangor, Malaysia \\ ${ }^{3}$ Department of Computer and \\ Communication Systems \\ Engineering, Faculty of Engineering, \\ University Putra Malaysia, UPM \\ Serdang 43400 Selangor, Malaysia \\ Full list of author information is \\ available at the end of the article
}

\begin{abstract}
The idea of multiple antenna arrays has evolved into multiple-input multiple-output (MIMO) system, which provides transmit and receive diversities. It increases robustness of the effect of multipath fading in wireless channels, besides yielding higher capacity, spectral efficiency and better bit error rate (BER) performance. The spatial diversity gain is obtained by transmitting or receiving multiple copies of a signal through different antennas to combat fading and improves the system BER performance. However, the computational complexity of MIMO system is inevitably increased. Space-time coding (STC) technique such as Alamouti's space-time block code (STBC) that combines coding, modulation and signal processing has been used to achieve spatial diversity. Vertical Bell Laboratories Layered Space-Time (V-BLAST) uses antenna arrays at both the transmitter and receiver to achieve spatial multiplexing gain. Independent data streams that share both frequency bands and time slots are transmitted from multiple antennas and jointly detected at the receiver. The theoretical capacity of V-BLAST increases linearly with the number of antennas in rich scattering environments. It's well-known that maximization of spatial diversity gain leads to degradation of spatial multiplexing gain or vice versa. In order to achieve spatial multiplexing and diversity gains simultaneously, the V-BLAST/STBC scheme has been introduced. This hybrid scheme increases MIMO system capacity and maintains reliable BER performance at the same time. However, both V-BLAST and STBC layers, in this hybid scheme, assume each other as an interferer. Thus, the symbols must be decoded with a suitable detection mechanism. In this paper, a new low complexity detection mechanism for V-BLAST/ STBC scheme based on QR decomposition, denoted as low complexity QR (LC-QR) decomposition, is presented. The performance of the proposed LC-QR decomposition detection mechanism in V-BLAST/STBC transceiver scheme is compared with other detection mechanisms such as ZF, MMSE and QR decomposition. It is shown that the BER performance in V-BLAST/STBC scheme is better than V-BLAST scheme while its system capacity is higher than orthogonal STBC scheme when the LC-QR decomposition detection mechanism is exploited. Moreover, the computational complexity of proposed LC-QR decomposition mechanism is significantly lower than other abovementioned detection mechanisms.
\end{abstract}

Keywords: MIMO; V-BLAST; STBC; MMSE; ZF; QR decomposition; Spatial diversity gain; Spatial multiplexing gain

\section{Springer}

(c) 2014 Chong et al.; licensee Springer. This is an Open Access article distributed under the terms of the Creative Commons Attribution License (http://creativecommons.org/licenses/by/2.0), which permits unrestricted use, distribution, and reproduction in any medium, provided the original work is properly cited. 


\section{Introduction}

Conventional single-input single-output (SISO) system, which is a wireless communication system with a single antenna at the transmitter and receiver, is vulnerable to multipath fading effect. Multipath is the arrival of the multiple copies of transmitting signal at the receiver through different angles, time delay or differing frequency (Doppler) shifts due to the scattering of electromagnetic waves. Each copy of the transmitted signal will experience differences in attenuation, delay and phase shift while travelling from the transmitter to the receiver. As a result, constructive or destructive interference is experienced at the receiver. The random fluctuation in signal level, known as fading [1,2], can severely affect the quality and reliability of wireless communication. Strong destructive interference will cause a deep fade and temporary failure of communication due to severe signal power attenuation. Moreover, the constraints posed by limited power, capacity and scarce spectrum make the design of SISO with high data rate and reliability extremely challenging.

The use of multiple antennas at the receiver and transmitter in a wireless network is rapidly superseding SISO to provide higher data rates at longer ranges especially for Long Term Evolution (LTE) systems [3] without consuming extra bandwidth or power. It is also a solution to the capacity limitation of the current wireless systems. The idea of multiple antennas has evolved into multiple-input multiple-output (MIMO) system, which provides transmit and receive diversities. It increases robustness of the effect of multi-path fading in wireless channels, besides yielding higher capacity, spectral efficiency and better bit error rate (BER) performance over conventional SISO systems in multipath fading environments [1,4]. However, the revolution of SISO to MIMO causes the computation complexity to be increased.

Vertical Bell Laboratories Layered Space-Time (V-BLAST) uses antenna arrays at both the transmitter and receiver to achieve spatial multiplexing gain. Independent data streams that share both frequency bands and time slots are transmitted from multiple antennas and jointly detected at the receiver. The theoretical capacity of V-BLAST increases linearly with the number of antennas in rich scattering environments [5]. The spatial diversity gain is obtained by transmitting or receiving multiple copies of a signal through different antennas. This scheme is designed to combat fading and improves the system BER performance. Space-time coding (STC) technique such as space-time trellis code (STTC) that combines coding, modulation and signal processing has been used to achieve spatial diversity [6]. It achieves maximum diversity and coding gain but the system computational complexity increases exponentially with transmission rate. Alamouti's space-time block code (STBC) [7] is another technique used to reduce the computational complexity in STTC. It supports linear decoding complexity for maximum-likelihood (ML) decoding. Orthogonal space-time block code (O-STBC), which is a generalization of the Alamouti's scheme to an arbitrary number of transmit antennas, was introduced in [8].

However, it was shown in [9] that there is a trade-off between spatial diversity gain and spatial multiplexing gain of MIMO systems. For instance, while the V-BLAST scheme increases spatial multiplexing gain, but it does not provide any spatial diversity gain. The V-BLAST scheme is more susceptible to multipath fading and noise compared to Alamouti's STBC scheme as there is no redundant information. Besides, the error propagation in V-BLAST detection causes BER performance degradation and 
limits the potential capacity of the V-BLAST scheme [10]. Although Alamouti's STBC provides full transmit and receive antenna diversity, the maximum code rate of one can be achieved for two transmit antennas only. For more than two antennas, the maximum possible code rate is $3 / 4$ [11], thus Alamouti's STBC could not satisfy the demand of the desired high system capacity in real time system with good quality of service (QoS) $[12,13]$. Therefore, in order to achieve spatial multiplexing and diversity gains simultaneously, the hybrid MIMO system has been introduced [14-16]. One of the hybrid MIMO systems is V-BLAST/STBC scheme. However, this hybrid scheme will further induce inevitably higher computational complexity in designing the system.

The V-BLAST/STBC scheme, which was introduced in [14] is a combination of the Alamouti's STBC and V-BLAST schemes. A number of research efforts on V-BLAST/ STBC scheme have been carried for MIMO system with the goal of maximizing the system capacity and reducing its computational complexity. The V-BLAST/STBC scheme improves the performance of MIMO by combining spatial multiplexing and diversity techniques together [17]. However, the spatially-multiplexed V-BLAST and STBC layers in the V-BLAST/STBC scheme assume each other as an interferer. Therefore, the transmitted symbols must be decoded with well-known detection mechanisms such as zero-forcing (ZF), minimum mean-squared error (MMSE) and QR decomposition which are employed in V-BLAST scheme [18]. Thus, the lowest computation complexity detection mechanism will be preferred. In this paper, a new detection mechanism based on QR decomposition, denoted as low complexity QR (LC-QR) decomposition, is presented. The $\mathrm{QR}$ decomposition of $A \times B$ channel matrix $\mathrm{H}$ is a factorization $\mathrm{H}=\mathrm{QR}$, where $\mathrm{Q}$ is $A \times B$ unitary matrix and $\mathrm{R}$ is $B \times B$ upper triangular matrix. The computational implementation of $\mathrm{QR}$ decomposition is less than ZF and MMSE [19], thus the computational complexity of V-BLAST/STBC scheme can be further reduced by using the proposed LC-QR decomposition detection mechanism.

The performance of V-BLAST/STBC transceiver scheme with proposed LC-QR decomposition mechanism is compared with V-BLAST and Alamouti's STBC schemes. It is shown that the BER performance of V-BLAST/STBC scheme is better than V-BLAST scheme while the system capacity of V-BLAST/STBC scheme is higher than STBC scheme when the LC-QR decomposition mechanism is exploited. Moreover, the computational complexity of proposed LC-QR decomposition mechanism is significantly lower than traditional ZF, MMSE and QR decomposition detection mechanisms. Since MIMO scheme is considered as the latest multiple access technique for the next generation human computer interaction (HCI) [20] or mobile computing devices, especially in LTE-Advanced system, higher computational complexity inherited with it is inevitable. Any computational complexity reduction mechanism can further reduce the computational cost and power consumption. Therefore, by using the proposed LC-QR decomposition detection mechanism in V-BLAST/STBC MIMO scheme the system performance is not only significantly improved but the computational complexity of the overall system is also significantly reduced.

The rest of the paper is organized as follows. In Section System models, an overview of the hybrid V-BLAST/STBC system model is presented with its traditional ZF, MMSE and $\mathrm{QR}$ decomposition decoder mechanisms in the sub-sections. Then, the proposed new LC-QR decomposition detection mechanism is introduced in Section LC-QR 
Decomposition Mechanism. In Section The Computational Complexity of LC-QR Decomposition Compared with ZF, MMSE and QR Decomposition Detection Mechanisms, the computational complexity comparison of LC-QR decomposition with other detection mechanisms is discussed. The system capacity and probability of error in V-BLAST/STBC scheme with LC-QR decomposition are examined in Sections System Capacity of V-BLAST/STBC Scheme with LC-QR Decomposition and Probability of Error in V-BLAST/STBC Scheme with LC-QR Decomposition respectively. Section Performance Evaluation of LC-QR Decomposition in V-BLAST/STBC Scheme illustrates the system performance of proposed LC-QR decomposition in V-BLAST/ STBC scheme. Finally, this paper concludes in Section Conclusions.

\section{System models}

The V-BLAST/STBC scheme, which was introduced in [14,17], is a combination of the Alamouti's STBC and V-BLAST schemes. It provides spatial diversity gain for high priority data and spatial multiplexing gain for low priority data simultaneously by partitioning a single data stream into two parallel sub-streams according to the data priority. The high priority data (e.g. frame header, I-frame, P-frame) is assigned to the STBC layer for extra protection while low priority data (e.g. B-frame, best-effort data) is sent to V-BLAST layer with higher capacity. Since high priority data is more important than low priority data, the corruption of high priority data will severely affect the real time service quality. For instance, error of any missing data in the first enhancement of P-frame layer is propagated to the subsequent P-frames, and it significantly degrades the perceived MPEG video quality. In contrast, any data loss in the B-frame layer affects only the corresponding frame, as it is not referred by other frames for decoding.

The block diagram of a V-BLAST/STBC transceiver model with $M(M \geq 3)$ transmit and $N(N \geq M-1)$ receive antennas is shown in Figure 1. A single main data stream is de-multiplexed into two sub-data streams according to the data priority. The high priority data is assigned to the STBC layer for extra gain while low priority data is sent to V-BLAST layer with higher capacity. As the Alamouti's STBC layer spans two

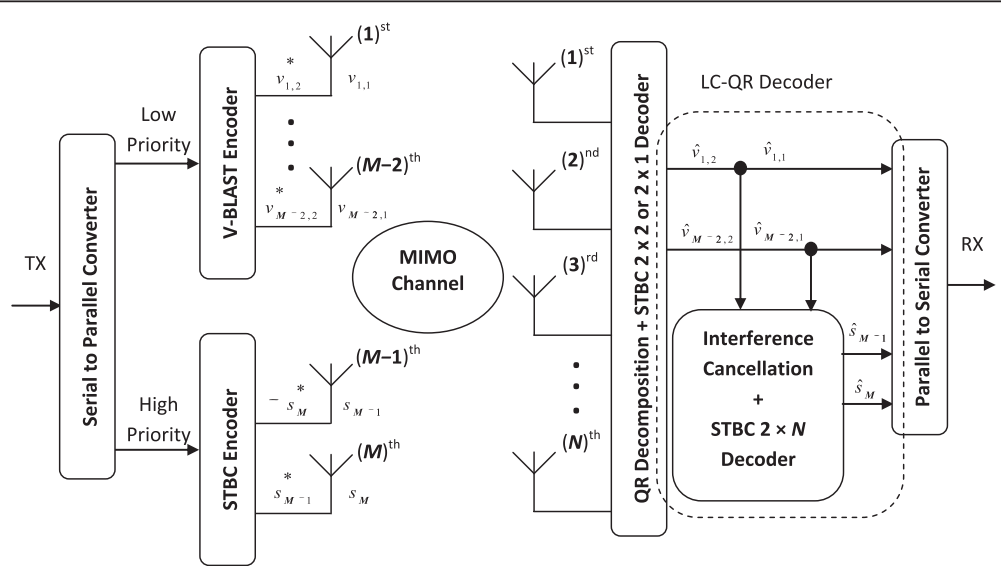

Figure 1 The proposed V-BLAST/STBC scheme consisting of a transmitter and a LC-QR decomposition receiver. 
symbol intervals, the V-BLAST/STBC scheme transmission matrix over two consecutive symbol periods is given as

$$
\mathrm{x}=\left[\begin{array}{cc}
v_{1,1} & v_{1,2}^{*} \\
\vdots & \vdots \\
v_{M-2,1} & v_{M-2,2}^{*} \\
s_{M-1} & -s_{M}^{*} \\
s_{M} & s_{M-1}^{*}
\end{array}\right] \begin{aligned}
& \text { V-BLAST } \\
& \text { Layer } \\
& \begin{array}{l}
\text { STBC } \\
\text { Layer }
\end{array}
\end{aligned}
$$

where "denotes the complex conjugate operation. The transmitted symbols are assumed to go through a Naftali channel coefficient matrix $\mathrm{H}$ where $\mathrm{H}$ is the $N \times M$ matrix as

$$
\mathbf{H}=\left[\begin{array}{cccc}
h_{1,1} & \cdots & h_{1, M-1} & h_{1, M} \\
h_{2,1} & \cdots & h_{2, M-1} & h_{2, M} \\
\vdots & \vdots & \vdots & \vdots \\
h_{N, 1} & \cdots & h_{N, M-1} & h_{N, M}
\end{array}\right]
$$

The complex channel coefficient of the Naftali channel model using random uniformly distributed phase and Rayleigh distributed magnitude can be described as

$$
h_{k}=N\left(0, \frac{\sigma_{k}^{2}}{2}\right)+j N\left(0, \frac{\sigma_{k}^{2}}{2}\right)
$$

where $\sigma_{k}^{2}=\sigma_{0}^{2} \exp \left(-k T_{s} / T_{r m s}\right), \sigma_{0}^{2}=1-\exp \left(-T_{s} / T_{r m s}\right)$, and $N\left(0, \frac{\sigma_{k}^{2}}{2}\right)$ is a zero mean Gaussian random variable with variance $\frac{\sigma_{k}^{2}}{2}$ produced by generating an $N(0,1)$ Gaussian random number and multiplying it by $\frac{\sigma_{k}}{\sqrt{2}}$. The $\sigma_{0}^{2}=1-\exp \left(-T_{s} / T_{r m s}\right)$ is chosen so that the condition $\sum_{k=0}^{k \max } \sigma_{k}^{2}=1$ is satisfied to ensure the same average received power.

The parameters $T_{s}$ and $T_{r m s}$ represent sampling period and delay spread of the channel respectively. The number of samples to be taken in the impulse response should ensure sufficient decay of the impulse response to avoid inter symbol interference.

At the receiver, the received matrix over two consecutive symbol periods can be expressed as $\mathrm{y}=\mathrm{Hx}+\mathrm{z}$ or

$$
\left[\begin{array}{cc}
y_{1,1} & y_{1,2} \\
y_{2,1} & y_{2,2} \\
\vdots & \vdots \\
y_{N, 1} & y_{N, 2}
\end{array}\right]=\left[\begin{array}{cccc}
h_{1,1} & \cdots & h_{1, M-1} & h_{1, M} \\
h_{2,1} & \cdots & h_{2, M-1} & h_{2, M} \\
\vdots & \vdots & \vdots & \vdots \\
h_{N, 1} & \cdots & h_{N, M-1} & h_{N, M}
\end{array}\right]\left[\begin{array}{cc}
v_{1,1} & v_{1,2}^{*} \\
\vdots & \vdots \\
v_{M-2,1} & v_{M-2,2}^{*} \\
s_{M-1} & -s_{M}^{*} \\
s_{M} & s_{M-1}^{*}
\end{array}\right]+\left[\begin{array}{cc}
z_{1,1} & z_{1,2} \\
z_{2,1} & z_{2,2} \\
\vdots & \vdots \\
z_{N, 1} & z_{N, 2}
\end{array}\right]
$$

where $\mathrm{y}$ is the $N \times 2$ received signal matrix and $\mathrm{x}$ is the $\mathrm{V}$-BLAST/STBC $M \times 2$ transmission matrix. $\mathrm{z}$ is the additive white Gaussian noise (AWGN) $N \times 2$ complex matrix with unit variance $\sigma^{2}$ and zero mean. For simplicity, it is assumed that the Naftali channel is constant across two consecutive symbol transmission periods, and hence the entries of $\mathrm{H}$ are average Naftali channel coefficients. The received matrix over two time 
intervals in (4) is re-arranged into a single vector to facilitate formulating the detection mechanism resulting as $V=\vec{H} S+N$ or

$$
\left[\begin{array}{c}
y_{1,1} \\
y_{2,1} \\
\vdots \\
y_{N, 1} \\
y_{1,2}^{*} \\
y_{2,2}^{*} \\
\vdots \\
y_{N, 2}^{*}
\end{array}\right]=\left[\begin{array}{cccccccc}
h_{1,1} & \cdots & h_{1, M-2} & 0 & \cdots & 0 & h_{1, M-1} & h_{1, M} \\
h_{2,1} & \cdots & h_{2, M-2} & 0 & \cdots & 0 & h_{2, M-1} & h_{2, M} \\
\vdots & \cdots & \vdots & \vdots & \cdots & \vdots & \vdots & \vdots \\
h_{N, 1} & \cdots & h_{N, M-2} & 0 & \cdots & 0 & h_{N, M-1} & h_{N, M} \\
0 & \cdots & 0 & h_{1,1}^{*} & \cdots & h_{1, M-2}^{*} & h_{1, M}^{*} & -h_{1, M-1}^{*} \\
0 & \cdots & 0 & h_{2,1}^{*} & \cdots & h_{2, M-2}^{*} & h_{2, M}^{*} & -h_{2, M-1}^{*} \\
\vdots & \cdots & \vdots & \vdots & \cdots & \vdots & \vdots & \vdots \\
0 & \cdots & 0 & h_{N, 1}^{*} & \cdots & h_{N, M-2}^{*} & h_{N, M}^{*} & -h_{N, M-1}^{*}
\end{array}\right]\left[\begin{array}{c}
v_{1,1} \\
\vdots \\
v_{M-2,1} \\
v_{1,2} \\
\vdots \\
v_{M-2,2} \\
s_{M-1} \\
s_{M}
\end{array}\right]+\left[\begin{array}{c}
z_{1,1} \\
z_{2,1} \\
\vdots \\
z_{N, 1} \\
z_{1,2}^{*} \\
z_{2,2}^{*} \\
\vdots \\
z_{N, 2}^{*}
\end{array}\right]
$$

From (5), it can be easily seen that the obtained system is equivalent to a spatial multiplexing scheme. The spatially multiplexed V-BLAST and STBC layers in the V-BLAST/ STBC scheme assume each other as interferer. Therefore, the transmitted symbols can be decoded with well-known detection techniques such as ZF, MMSE and QR decomposition which are employed in V-BLAST scheme [18]. The ZF and MMSE techniques involve the computation of Moore-Penrose pseudo-inverse of a matrix with cubic computational complexity. Beside MMSE and ZF, QR decomposition is also a common signal processing technique for MIMO detection [21]. The QR decomposition of $A \times B$ channel matrix $\mathrm{H}$ is a factorization $\mathrm{H}=\mathrm{QR}$, where $\mathrm{Q}$ is $A \times B$ unitary matrix and $\mathrm{R}$ is $B \times$ $B$ upper triangular matrix. The computational implementation of $\mathrm{QR}$ decomposition is less than ZF and MMSE [19], thus the computational complexity of V-BLAST/STBC scheme can be reduced using QR decomposition. The brief overview of ZF, MMSE and QR decomposition decoder are presented in following sub-sections.

\section{Zero-forcing (ZF) decoder}

A ZF V-BLAST/STBC receiver architecture is given in $[18,22]$ where all the undesired sub-data streams are nulled by linear weighting receive vector $V$ at each detecting step. The ZF decoded symbol $\hat{S}_{i}^{\mathrm{ZF}}$ of the $i$-th sub-stream is calculated by multiplying the $i$-th row of the equalizer filter matrix $W$ with the receive vector, and $\hat{S}_{i}^{\mathrm{ZF}}$ is given by

$$
\hat{S}_{i}^{\mathrm{ZF}}=(W)_{i} V
$$

where $(W)_{i}$ represents the $i$-th row of matrix $W$ corresponds the criterion in use. The equalizer filter matrix $W$ is then given by

$$
W=\left(\vec{H}^{H} \vec{H}\right)^{-1} \vec{H}^{H}
$$

In an orthogonal channel matrix, ZF is identical to ML. However, in general ZF leads to noise amplification, which is especially observed in systems with the same number of transmit and receive antennas.

\section{Minimum mean squared error (MMSE) decoder}

In a MMSE decoder, each received sub-data stream is the superposition of the desired signal [22]. The undesired sub-data streams are considered as interference. At each decoding step, all undesired sub-streams are nulled by linear weighting receive vector $V$. The MMSE decoded symbol $\hat{S}_{i}^{\mathrm{MMSE}}$ of the $i$-th sub-stream is calculated by multiplying the 
$i$-th row of the equalizer filter matrix $D$ with the receive vector. Hence, $\hat{S}_{i}^{\text {MMSE }}$ for MMSE criterion is given as

$$
\hat{S}_{i}^{\mathrm{MMSE}}=(D)_{i} V
$$

where $(D)_{i}$ represents the $i$-th row of matrix $D$ corresponding to the criterion in use. The equalizer filter matrix $D$ is then given by

$$
D=\left(\vec{H}^{H} \vec{H}+\sigma^{2} I_{2(M-1)}\right)^{-1} \vec{H}^{H}
$$

where $I_{2(M-1)}$ is the $2(M-1) \times 2(M-1)$ identity matrix. The MMSE detector takes the noise term into account and leads to performance enhancement compared to ZF consequently.

\section{QR decomposition decoder}

The QR decomposition decoder is applied to the equivalent channel matrix $\vec{H}$ to start the V-BLAST/STBC detection mechanism. QR decomposition is performed with $\vec{H}=\vec{Q}$ $\vec{R}$ which is given as

$$
\vec{H}=\underbrace{\left[\begin{array}{ccc}
q_{1,1} & \cdots & q_{1,2 M-2} \\
q_{2,1} & \cdots & q_{2,2 M-2} \\
\vdots & \cdots & \vdots \\
q_{2 N-1,1} & \cdots & q_{2 N-1,2 M-2} \\
q_{2 N, 1} & \cdots & q_{2 N, 2 M-2}
\end{array}\right]}_{\vec{Q}} \underbrace{\left[\begin{array}{cccccc}
r_{1,1} & r_{1,2} & \cdots & \cdots & r_{1,2 M-3} & r_{1,2 M-2} \\
0 & r_{2,2} & \cdots & \cdots & r_{2,2 M-3} & r_{2,2 M-2} \\
0 & 0 & \vdots & \vdots & \vdots & \vdots \\
0 & 0 & \vdots & \vdots & \vdots & \vdots \\
0 & 0 & \cdots & \cdots & r_{2 M-3,2 M-3} & r_{2 M-3,2 M-2} \\
0 & 0 & \cdots & \cdots & 0 & r_{2 M-2,2 M-2}
\end{array}\right]}_{\vec{R}}
$$

where $\vec{Q}$ is a unit norm orthogonal columns $2 N \times 2(M-1)$ matrix and $\vec{R}$ is a $2(M-1) \times$ $2(M-1)$ upper triangular square matrix. The QR factorization can be calculated with the Householder reflection [23]. Left-multiplying (5) by $\vec{Q} H$ results $\vec{V}=\vec{R} S+\vec{N}$ or

$$
\left[\begin{array}{l}
\vec{y}_{1,1} \\
\vec{y}_{2,1} \\
\vec{y}_{3,1} \\
\vec{y}_{4,1} \\
\vec{y}_{5,1} \\
\vec{y}_{6,1}
\end{array}\right]=\left[\begin{array}{cccccc}
r_{1,1} & r_{1,2} & \cdots & \cdots & r_{1,2 M-3} & r_{1,2 M-2} \\
0 & r_{2,2} & \cdots & \cdots & r_{2,2 M-3} & r_{2,2 M-2} \\
0 & 0 & \vdots & \vdots & \vdots & \vdots \\
0 & 0 & \vdots & \vdots & \vdots & \vdots \\
0 & 0 & \cdots & \cdots & r_{2 M-3,2 M-3} & r_{2 M-3,2 M-2} \\
0 & 0 & \cdots & \cdots & 0 & r_{2 M-2,2 M-2}
\end{array}\right]\left[\begin{array}{c}
v_{1,1} \\
\vdots \\
v_{M-2,1} \\
v_{1,2} \\
\vdots \\
v_{M-2,2} \\
s_{M-1} \\
s_{M}
\end{array}\right]+\left[\begin{array}{c}
\vec{z}_{1,1} \\
\vec{z}_{2,1} \\
\vec{z}_{3,1} \\
\vec{z}_{4,1} \\
\vec{z}_{5,1} \\
\vec{z}_{6,1}
\end{array}\right]
$$

where $\vec{V}=\vec{Q} H V$ and $\vec{N}=\vec{Q} H N$. From (11), $s_{M}$ is decoded first, followed by $s_{M-1}, \ldots$, and finally $v_{1,1}$.

\section{LC-QR decomposition mechanism}

The computational complexity of ZF, MMSE and QR decomposition with $\vec{H}$, which have been discussed in the previous section, is high because of the equivalent channel matrix $\vec{H}$ with dimension $2 N \times 2(M-1)$ in (5). Therefore, a low complexity V-BLAST/ 
STBC detection mechanism with QR decomposition, denoted as LC-QR decomposition, is proposed. Instead of decoding the transmitted symbols with the equivalent channel matrix $\vec{H}$ with dimension $2 N \times 2(M-1)$, the original channel matrix $\mathrm{H}$ in (2) with dimension $N \times M$ is utilized. If $N<M-1$, the LC-QR decomposition mechanism is invalid and not applicable. The flow chart of LC-QR decomposition mechanism is shown in Figure 2.

Case A: $N$ receive antenna is greater than or equal to $M$ transmit antenna $(N \geq M)$

The five steps of the LC-QR decomposition mechanism for case $\mathrm{A}(N \geq M)$ are described as follows.

Step 1: Application of $\mathrm{QR}$ decomposition to the channel matrix $\mathrm{H}$

The QR decomposition is applied to the channel matrix $\mathrm{H}$ with dimension $N \times M$ to start the V-BLAST/STBC detection mechanism. The QR decomposition is performed with $\mathrm{H}=\mathrm{QR}$ by

$$
\mathbf{H}=\underbrace{\left[\begin{array}{cccc}
\mathrm{Q}_{1,1} & \mathrm{Q}_{1,2} & \cdots & \mathrm{Q}_{1, M} \\
\mathrm{Q}_{2,1} & \mathrm{Q}_{2,2} & \cdots & \mathrm{Q}_{2, M} \\
\vdots & \vdots & \vdots & \vdots \\
\mathrm{Q}_{N, 1} & \mathrm{Q}_{N, 2} & \cdots & \mathrm{Q}_{N, M}
\end{array}\right]}_{\mathrm{Q}} \underbrace{\left[\begin{array}{cccc}
\mathrm{R}_{1,1} & \mathrm{R}_{1,2} & \cdots & \mathrm{R}_{1, M} \\
0 & \mathrm{R}_{2,2} & \cdots & \mathrm{R}_{2, M} \\
0 & 0 & \cdots & \vdots \\
0 & 0 & 0 & \mathrm{R}_{M, M}
\end{array}\right]}_{\mathrm{R}}
$$

where $\mathrm{Q}$ is a unit norm orthogonal columns $N \times M$ matrix and $\mathrm{R}$ is a $M \times M$ upper triangular square matrix.

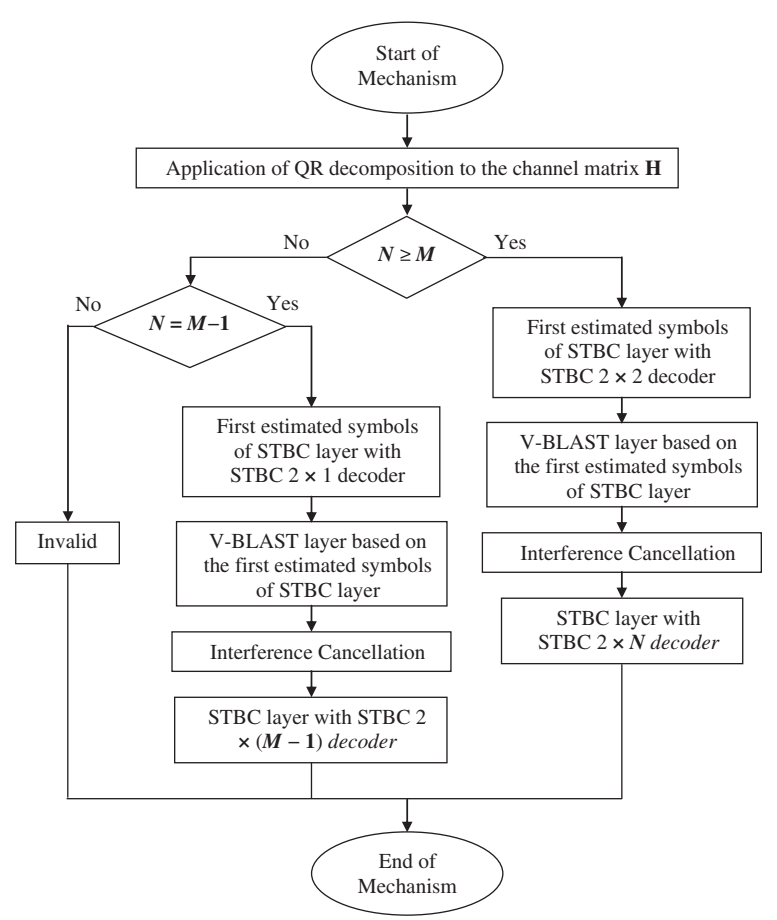

Figure 2 The flow chart of LC-QR decomposition mechanism. 
Left-multiplying (4) by $\mathrm{Q}^{H}$, we get $\tilde{\mathrm{y}}=\mathbf{R} \mathrm{x}+\tilde{\mathrm{z}}$ or

$$
\left[\begin{array}{cc}
\tilde{y}_{1,1} & \tilde{y}_{1,2} \\
\tilde{y}_{2,1} & \tilde{y}_{2,2} \\
\vdots & \vdots \\
\tilde{y}_{M, 1} & \tilde{y}_{M, 2}
\end{array}\right]=\left[\begin{array}{cccc}
\mathrm{R}_{1,1} & \mathrm{R}_{1,2} & \cdots & \mathrm{R}_{1, M} \\
0 & \mathrm{R}_{2,2} & \cdots & \mathrm{R}_{2, M} \\
0 & 0 & \cdots & \vdots \\
0 & 0 & 0 & \mathrm{R}_{M, M}
\end{array}\right]\left[\begin{array}{cc}
v_{1,1} & v_{1,2}^{*} \\
\vdots & \vdots \\
v_{M-2,1} & v_{M-2,2}^{*} \\
s_{M-1} & -s_{M}^{*} \\
s_{M} & s_{M-1}^{*}
\end{array}\right]+\left[\begin{array}{cc}
\tilde{z}_{1,1} & \tilde{z}_{1,2} \\
\tilde{z}_{2,1} & \tilde{z}_{2,2} \\
\vdots & \vdots \\
\tilde{z}_{M, 1} & \tilde{z}_{M, 2}
\end{array}\right]
$$

where $\tilde{\mathrm{y}}=\mathrm{Q}^{H} \mathrm{y}$ and $\tilde{\mathrm{z}}=\mathrm{Q}^{H} \mathrm{z}$.

Step 2: Calculate the first estimated symbols of STBC layer with STBC $2 \times 2$ decoder

In $\mathrm{QR}$ decomposition, the triangular properties of $\mathrm{R}$ allow the sequence of transmitted symbols to be recovered by simple backward substitution with cancellation. If decision error exists in the first detected sub-data streams, the decision feedbacks will significantly increase the probability of error in decoding the subsequent sub-data streams. Therefore, the first decoding decision is made based on STBC layer first so as to decrease the probability of error in decoding the V-BLAST layer. In step 2, $\mathrm{R}_{M-1, M-1}$, $\mathrm{R}_{M-1, M}$ and $\mathrm{R}_{M, M}$ of upper triangular square matrix $\mathrm{R}$ are utilized to calculate the first estimated symbols of the STBC layer with STBC $2 \times 2$ decoder. These parameters are derived as

$$
\begin{aligned}
& \tilde{s}_{M-1}=Q\left(\mathrm{R}_{M-1, M-1}^{*} \tilde{\mathrm{Y}}_{M-1,1}+\mathrm{R}_{M-1, M} \tilde{\mathrm{Y}}_{M-1,2}^{*}+\mathrm{R}_{M, M} \tilde{\mathrm{Y}}_{M, 2}^{*}\right) \\
& \tilde{s}_{M}=Q\left(\mathrm{R}_{M-1, M}^{*} \tilde{\mathrm{Y}}_{M-1,1}-\mathrm{R}_{M-1, M-1} \tilde{\mathrm{Y}}_{M-1,2}^{*}+\mathrm{R}_{M, M}^{*} \tilde{\mathrm{Y}}_{M, 1}\right)
\end{aligned}
$$

where $Q(\cdot)$ denotes the quantization (slicing) operation appropriate to the constellation in use.

Step 3: Decode the data symbols of V-BLAST layer based on the first estimated symbols of STBC layer

The V-BLAST layer data symbols are decoded with the properties of upper triangular square matrix R in (13) and first estimated symbols of STBC layer. These layer symbols in the first and second time slot are then derived in a successive way as

$$
\begin{aligned}
& \hat{v}_{i, 1}=Q\left(\frac{\tilde{\mathrm{y}}_{i, 1}-\left(\sum_{j=i+1}^{M-2} \mathrm{R}_{i, j} \hat{v}_{j, 1}+\mathrm{R}_{i, M-1} \tilde{s}_{M-1}+\mathrm{R}_{i, M} \tilde{s}_{M}\right)}{\mathrm{R}_{i, i}}\right) \\
& \hat{v}_{i, 2}=Q\left(\left(\frac{\tilde{\mathrm{y}}_{i, 2}-\left(\sum_{j=i+1}^{M-2} \mathrm{R}_{i, j} \hat{v}_{j, 2}+\mathrm{R}_{i, M-1}\left(-\tilde{s}_{M}^{*}\right)+\mathrm{R}_{i, M}\left(\tilde{s}_{M-1}^{*}\right)\right)}{\mathrm{R}_{i, i}}\right)\right)^{*}
\end{aligned}
$$

\section{Step 4: Interference cancellation}

A new modified received $N \times 2$ matrix, $\mathrm{s}$ is created by subtracting the data symbols of $\mathrm{V}$-BLAST layer from the original received matrix $y$ in this step, which results in the following matrix 


$$
\left[\begin{array}{cc}
s_{1,1} & s_{1,2} \\
s_{2,1} & s_{2,2} \\
\vdots & \vdots \\
s_{N, 1} & s_{N, 2}
\end{array}\right]=\left[\begin{array}{cc}
y_{1,1} & y_{1,2} \\
y_{2,1} & y_{2,2} \\
\vdots & \vdots \\
y_{N, 1} & y_{N, 2}
\end{array}\right]-\left[\begin{array}{ccc}
h_{1,1} & \cdots & h_{1, M-2} \\
h_{2,1} & \cdots & h_{2, M-2} \\
\vdots & \vdots & \vdots \\
h_{N, 1} & \cdots & h_{N, M-2}
\end{array}\right]\left[\begin{array}{cc}
\hat{v}_{1,1} & \hat{v}_{1,2}^{*} \\
\vdots & \vdots \\
\hat{v}_{M-2,1} & \hat{v}_{M-2,2}^{*}
\end{array}\right]
$$

Step 5: Decode the data symbols of STBC layer from the new modified received matrix with STBC $2 \times N$ decoder

The data symbols of STBC layer are decoded from the new modified received matrix $\mathrm{s}$ with $\mathrm{STBC} 2 \times N$ decoder and are given by

$$
\begin{aligned}
& \hat{s}_{M-1}=Q\left(\sum_{k=1}^{N}\left(h_{k, M-1}^{*} s_{k, 1}+h_{k, M} s_{k, 2}^{*}\right)\right) \\
& \hat{s}_{M}=Q\left(\sum_{k=1}^{N}\left(h_{k, M}^{*} s_{k, 1}-h_{k, M-1} s_{k, 2}^{*}\right)\right)
\end{aligned}
$$

Case B: $N$ receive antenna is less than $M$ transmit antenna by $1(N=M-1)$

The five steps of the LC-QR mechanism for case $\mathrm{B}(N=M-1)$ are described as follows.

Step 1: Application of $\mathrm{QR}$ decomposition to the channel matrix $\mathrm{H}$

The QR decomposition is applied to the channel matrix $\mathrm{H}$ with dimension $(M-1) \times$ $M$ to start the V-BLAST/STBC detection mechanism. The QR decomposition is performed with $\mathrm{H}=\mathrm{QR}$ by

$$
\mathbf{H}=\underbrace{\left[\begin{array}{cccc}
\mathrm{Q}_{1,1} & \mathrm{Q}_{1,2} & \cdots & \mathrm{Q}_{1, M-1} \\
\mathrm{Q}_{2,1} & \mathrm{Q}_{2,2} & \cdots & \mathrm{Q}_{2, M-1} \\
\vdots & \vdots & \vdots & \vdots \\
\mathrm{Q}_{M-1,1} & \mathrm{Q}_{M-1,2} & \cdots & \mathrm{Q}_{M-1, M-1}
\end{array}\right]}_{\mathrm{Q}} \underbrace{\left[\begin{array}{ccccc}
\mathrm{R}_{1,1} & \mathrm{R}_{1,2} & \cdots & \mathrm{R}_{1, M-1} & \mathrm{R}_{1, M} \\
0 & \mathrm{R}_{2,2} & \cdots & \mathrm{R}_{2, M-1} & \mathrm{R}_{2, M} \\
0 & 0 & \cdots & \vdots & \vdots \\
0 & 0 & 0 & \mathrm{R}_{M-1, M-1} & \mathrm{R}_{M-1, M}
\end{array}\right]}_{\mathrm{R}}
$$

where $\mathrm{Q}$ is a unit norm orthogonal columns $(M-1) \times(M-1)$ matrix and $\mathrm{R}$ is a $(M-1) \times$ $M$ upper triangular square matrix. Left-multiplying (4) by $\mathrm{Q}^{H}$, we get $\tilde{\mathrm{y}}=\mathrm{Rx}+\tilde{\mathrm{z}}$ or

$$
\begin{aligned}
{\left[\begin{array}{cc}
\tilde{y}_{1,1} & \tilde{y}_{1,2} \\
\tilde{y}_{2,1} & \tilde{y}_{2,2} \\
\vdots & \vdots \\
\tilde{y}_{M-1,1} & \tilde{y}_{M-1,2}
\end{array}\right]=} & {\left[\begin{array}{ccccc}
\mathrm{R}_{1,1} & \mathrm{R}_{1,2} & \cdots & \mathrm{R}_{1, M-1} & \mathrm{R}_{1, M} \\
0 & \mathrm{R}_{2,2} & \cdots & \mathrm{R}_{2, M-1} & \mathrm{R}_{2, M} \\
0 & 0 & \cdots & \vdots & \vdots \\
0 & 0 & 0 & \mathrm{R}_{M-1, M-1} & \mathrm{R}_{M-1, M}
\end{array}\right]\left[\begin{array}{cc}
v_{1,1} & v_{1,2}^{*} \\
\vdots & \vdots \\
v_{M-2,1} & v_{M-2,2}^{*} \\
s_{M-1} & -s_{M}^{*} \\
s_{M} & s_{M-1}^{*}
\end{array}\right] } \\
& +\left[\begin{array}{ccc}
\tilde{z}_{1,1} & \tilde{z}_{1,2} \\
\tilde{z}_{2,1} & \tilde{z}_{2,2} \\
\vdots & \vdots \\
\tilde{z}_{M-1,1} & \tilde{z}_{M-1,2}
\end{array}\right]
\end{aligned}
$$

where $\tilde{\mathrm{y}}=\mathrm{Q}^{H} \mathrm{y}$ and $\tilde{\mathrm{z}}=\mathrm{Q}^{H} \mathrm{z}$.

Step 2: Calculate the first estimated symbols of the STBC layer with STBC $2 \times 1$ decoder 
In QR decomposition, the triangular properties of $\mathrm{R}$ allow the sequence of transmitted symbols to be recovered by simple backward substitution with cancellation. If decision error exists in the first detected sub-data streams, the decision feedbacks will significantly increase the probability of error in decoding the subsequent sub-data streams. Therefore, the first decoding decision is made based on STBC layer first so as to decrease the probability of error in decoding the V-BLAST layer. In step $2, \mathrm{R}_{M-1, M-1}$ and $\mathrm{R}_{M-1, M}$ of upper triangular square matrix $\mathrm{R}$ are utilized to calculate the first estimated symbols of the STBC layer with STBC $2 \times 1$ decoder. These parameters are derived as

$$
\begin{aligned}
& \tilde{s}_{M-1}=Q\left(\mathrm{R}_{M-1, M-1}^{*} \tilde{\mathrm{Y}}_{M-1,1}+\mathrm{R}_{M-1, M} \tilde{\mathrm{Y}}_{M-1,2}^{*}\right) \\
& \tilde{s}_{M}=Q\left(\mathrm{R}_{M-1, M}^{*} \tilde{\mathrm{y}}_{M-1,1}-\mathrm{R}_{M-1, M-1} \tilde{\mathrm{y}}_{M-1,2}^{*}\right)
\end{aligned}
$$

where $Q(\cdot)$ denotes the quantization (slicing) operation appropriate to the constellation in use.

Step 3: Decode the data symbols of V-BLAST layer based on the first estimated symbols of STBC layer

The V-BLAST layer data symbols are decoded with the properties of upper triangular square matrix $\mathrm{R}$ in (22) and first estimated symbols of STBC layer. These layer symbols in the first and second time slot are then derived in a successive way as

$$
\begin{aligned}
& \hat{v}_{i, 1}=Q\left(\frac{\tilde{\mathrm{y}}_{i, 1}-\left(\sum_{j=i+1}^{M-2} \mathrm{R}_{i, j} \hat{v}_{j, 1}+\mathrm{R}_{i, M-1} \tilde{s}_{M-1}+\mathrm{R}_{i, M} \tilde{s}_{M}\right)}{\mathrm{R}_{i, i}}\right) \\
& \hat{v}_{i, 2}=Q\left(\left(\frac{\tilde{\mathrm{y}}_{i, 2}-\left(\sum_{j=i+1}^{M-2} \mathrm{R}_{i, j} \hat{v}_{j, 2}+\mathrm{R}_{i, M-1}\left(-\tilde{s}_{M}^{*}\right)+\mathrm{R}_{i, M}\left(\tilde{s}_{M-1}^{*}\right)\right)}{\mathrm{R}_{i, i}}\right)\right)
\end{aligned}
$$

\section{Step 4: Interference Cancellation}

A new modified received $(M-1) \times 2$ matrix, $\mathrm{s}$ is created by subtracting the data symbols of V-BLAST layer from the original received matrix y in this step, which results in the following matrix

$$
\left[\begin{array}{cc}
s_{1,1} & s_{1,2} \\
s_{2,1} & s_{2,2} \\
\vdots & \vdots \\
s_{M-1,1} & s_{M-1,2}
\end{array}\right]=\left[\begin{array}{cc}
y_{1,1} & y_{1,2} \\
y_{2,1} & y_{2,2} \\
\vdots & \vdots \\
y_{M-1,1} & y_{M-1,2}
\end{array}\right]-\left[\begin{array}{ccc}
h_{1,1} & \cdots & h_{1, M-2} \\
h_{2,1} & \cdots & h_{2, M-2} \\
\vdots & \vdots & \vdots \\
h_{M-1,1} & \cdots & h_{M-1, M-2}
\end{array}\right]\left[\begin{array}{cc}
\hat{v}_{1,1} & \hat{v}_{1,2}^{*} \\
\vdots & \vdots \\
\hat{v}_{M-2,1} & \hat{v}_{M-2,2}^{*}
\end{array}\right]
$$

Step 5: Decode the data symbols of STBC layer from the new modified received matrix with STBC $2 \times(M-1)$ decoder 
The data symbols of STBC layer are decoded from the new modified received matrix $\mathrm{s}$ with STBC $2 \times(M-1)$ decoder and are given by

$$
\begin{aligned}
& \hat{s}_{M-1}=Q\left(\sum_{k=1}^{M-1}\left(h_{k, M-1}^{*} s_{k, 1}+h_{k, M} s_{k, 2}^{*}\right)\right) \\
& \hat{s}_{M}=Q\left(\sum_{k=1}^{M-1}\left(h_{k, M}^{*} s_{k, 1}-h_{k, M-1} s_{k, 2}^{*}\right)\right)
\end{aligned}
$$

\section{The computational complexity of LC-QR decomposition compared with ZF, MMSE and QR decomposition detection mechanisms}

The channel matrix $\mathrm{H}$ with dimension $N \times M$ is used to analyze the computational complexity of the LC-QR decomposition. The equivalent channel matrix $\vec{H}$ with dimension $2 N \times 2(M-1)$ is used to analyze the computational complexity of $\mathrm{ZF}$,

MMSE and QR decomposition. It is observed that there are zeros in channel matrix $\vec{H}$, therefore the multiplication and addition with zero are not taken into account in ZF and MMSE complexity calculation. The detail of computational complexity of each mechanism is presented in the following sub-sections.

\section{Zero-Forcing (ZF) with $\vec{H}$}

From (7), the process to calculate the ZF equalizer filter matrix $W$ is divided into four steps.

Step 1 involves multiplication of $\vec{H}^{H}$ with $\vec{H}$, it requires $8 N(M-1)^{2}$ multiplications and $4(2 N-1)(M-1)^{2}$ additions, so the number of complex arithmetic operations in step 1 is $4(4 N-1)(M-1)^{2}$.

Step 2 involves Gaussian elimination matrix inversion of $\vec{H}^{H} \vec{H}$. According to [23], the computational complexity of Gaussian elimination matrix inversion with dimension a $\times$ b matrix is $O\left(\mathrm{ab}^{2}\right)$. Since the dimension of matrix $\vec{H}^{H} \vec{H}$ is $2(M-1) \times 2(M-1)$, thus the number of complex arithmetic operations in step 2 is $8(M-1)^{3}$.

Step 3 performs multiplication of $\left(\vec{H}^{H} \vec{H}\right)^{-1}$ with $\vec{H}^{H}$, it requires $8 N(M-1)^{2}$ multiplications and $8 N(M-1)^{2}-4 N(M-1)$ additions, then the number of complex arithmetic operations in step 3 is $16 N(M-1)^{2}-4 N(M-1)$.

In step 4 , the decoded symbols with ZF mechanism are calculated by multiplying equalizer filter matrix $W$ with the receive vector $V$ and it needs $4 N(M-1)$ multiplications and $2(2 N-1)(M-1)$ additions. So, the number of complex arithmetic operations in step 4 is $2(4 N-1)(M-1)$.

Finally, the total complex arithmetic operation of ZF V-BLAST/STBC is $8(M-1)^{3}+$ $4(8 N-1)(M-1)^{2}+2(2 N-1)(M-1)$.

\section{Minimum mean-squared error (MMSE) with $\vec{H}$}

From (9), the process to calculate the MMSE equalizer filter matrix $D$ is divided into five steps. 
Step 1 involves multiplication of $\vec{H}^{H}$ with $\vec{H}$, it requires $8 N(M-1)^{2}$ multiplications and $4(2 N-1)(M-1)^{2}$ additions, so the number of complex arithmetic operations in step 1 is $4(4 N-1)(M-1)^{2}$.

Step 2 performs the addition of $\vec{H}^{H} \vec{H}$. with $\sigma^{2} I_{2(M-1)}$, it requires $I_{2(M-1)}$ additions, and then the number of complex arithmetic operations in step 2 is $I_{2(M-1)}$.

Step 3 involves Gaussian elimination matrix inversion of $\left(\vec{H}^{H} \vec{H}+\sigma^{2} I_{2(M-1)}\right)$. Since the dimension of matrix $\left(\vec{H}^{H} \vec{H}+\sigma^{2} I_{2(M-1)}\right)$ is $2(M-1) \times 2(M-1)$, thus the number of complex arithmetic operations in step 3 is $8(M-1)^{3}$ [23].

Step 4 performs multiplication of $\left(\vec{H}^{H} \vec{H}+\sigma^{2} I_{2(M-1)}\right)^{-1}$ with $\vec{H}^{H}$, it requires $8 N$ $(M-1)^{2}$ multiplications and $8 N(M-1)^{2}-4 N(M-1)$ additions, then the number of complex arithmetic operations in step 4 is $16 N(M-1)^{2}-4 N(M-1)$.

In step 5 , the decoded symbols with MMSE mechanism are calculated by multiplying equalizer filter matrix $D$ with the receive vector $V$ and it needs $4 N(M-1)$ multiplications and $2(2 N-1)(M-1)$ additions. So, the number of complex arithmetic operations in step 5 is $2(4 N-1)(M-1)$.

Finally, the total complex arithmetic operation of MMSE V-BLAST/STBC is 8 $(M-1)^{3}+4(8 N-1)(M-1)^{2}+4 N(M-1)$.

\section{Conventional QR decomposition with $\vec{H}$}

Step 1 involves application of QR decomposition to the channel matrix $\vec{H}$ and multiplication of $\vec{Q} H$ with $V$. According to [23], the computational complexity of QR decomposition by Householder reflection with size $\mathrm{a} \times \mathrm{b}$ matrix is $O\left(a b^{2}-b^{3} / 3\right)$. Since the dimension of channel matrix $\vec{H}$ is $2 N \times 2(M-1)$, thus the number of complex arithmetic operations for the QR decomposition of $\vec{H}$ being $8 N(M-1)^{2}-(8 / 3)(M-1)^{3}$. Besides, for the multiplication of $\vec{Q} H$ with $V$, it needs $2 N \times 2(M-1)$ multiplications and $(2 N-1) \times$ $2(M-1)$ additions. Therefore, the number of complex arithmetic operations in step 1 is 8 $N(M-1)^{2}-(8 / 3)(M-1)^{3}+2(4 N-1) \times(M-1)$.

Step 2 decodes the transmitted symbols with backward substitution with cancellation; it requires $(M-2) \times(2 M-3)$ additions, $2(M-1)-1$ subtractions, $(2 M-3) \times(M-1)$ multiplications and $2(M-1)$ divisions.

Finally, the total complex arithmetic operation of conventional QR decomposition is $8 N(M-1)^{2}-(8 / 3)(M-1)^{3}+(2 M-3)^{2}+2(M-1)(4 N+1)-1$.

\section{LC-QR decomposition with $\mathrm{H}$}

Step 1 of LC-QR decomposition involves application of QR decomposition to the channel matrix $\mathrm{H}$ and multiplication of $\mathrm{Q}^{H}$ with y. Since the dimension of channel matrix $\mathrm{H}$ is $N \times M$, thus the number of complex arithmetic operations for the QR decomposition of $\mathrm{H}$ is $N M^{2}-M^{3} / 3$. Besides, for the multiplication of $\mathrm{Q}^{H}$ with you, it needs $N \times M$ multiplications and $(N-1) \times M$ additions. Therefore, the number of complex arithmetic operations in step 1 is $N M^{2}-M^{3} / 3+M(2 N-1)$. 
Step 2 of LC-QR decomposition calculates the first estimate symbols of the STBC layer with STBC $2 \times 2$ decoder. It needs six multiplications, three additions and one subtraction. So, the number of complex arithmetic operations in step 2 is ten.

Step 3 of the LC-QR decomposition decodes the data symbols of V-BLAST layer based on the first estimate symbols of STBC layer, so it requires $(M-2)(M-1)$ additions, $2(M-2)$ subtractions, $(M-2)(M+1)$ multiplications and $2(M-2)$ divisions. Therefore, the number of complex arithmetic operations in step 3 is $2(M-2)(M+2)$.

Step 4 of LC-QR decomposition involves interference cancellation, thus it requires 2 $N(M-2)$ multiplications, $2 N(M-3)$ additions and $2 N$ subtractions. Therefore, the number of complex arithmetic operations in step 4 is $4 N(M-2)$.

In Step 5, it decodes the data symbols of the STBC layer from the new modified received matrix s with STBC $2 \times N$ decoder, it requires $4 N$ multiplications, $N+2(N-1)$ additions and $N$ subtractions. Therefore, the number of complex arithmetic operations in step 5 is $2(4 N-1)$.

Finally, the total complex arithmetic operation of LC-QR is $N M^{2}-M^{3} / 3+(M+2)$ $(2 N-1)+2(M-2)(M+2)+4 N(M-1)+10$.

The comparison of number of complex arithmetic operations and reduction of computational complexity for ZF, MMSE, QR decomposition with $\vec{H}$ and LC-QR decomposition detection mechanism with $\mathrm{H}$ is shown in Tables 1 and 2 respectively. From Table 2, it can be observed that the proposed LC-QR decomposition detection mechanism significantly reduces the arithmetic complexity compared to ZF, MMSE and QR decomposition mechanisms.

\section{System capacity of V-BLAST/STBC scheme with LC-QR decomposition}

The instantaneous capacity of an orthogonal STBC of rate $r_{c}$ and $M$ transmit antennas, denoted as $\boldsymbol{C}_{S T B C}$, is given by [24]

$$
C_{S T B C}=\boldsymbol{r}_{c} \log _{2}\left(1+\frac{\rho}{M}\|\mathbf{H}\|^{2}\right)
$$

where $\rho$ is the signal-to-noise ratio (SNR) per receive antenna. According to (14) and (15), only $\mathrm{R}_{M-1, M-1}, \mathrm{R}_{M-1, M}$ and $\mathrm{R}_{M, M}$ of upper triangular square matrix $\mathrm{R}$ are required to decode the $\mathrm{STBC}$ layer, thus the instantaneous system capacity of the STBC layer of the LC-QR with $r_{c}=1$ and $M=4$, denoted as $C_{P_{-} \text {STBC }}$, is [25]:

$$
C_{P_{-} S T B C}=\log _{2}\left(1+\frac{\rho}{4}\left(\left|\mathrm{R}_{M-1, M-1}\right|^{2}+\left|\mathrm{R}_{M-1, M}\right|^{2}+\left|\mathrm{R}_{M, M}\right|^{2}\right)\right)
$$

From the procedure of decoding V-BLAST layer symbols in (16) and (17), the SNR of every V-BLAST channel can be determined. The received sub-data streams of $\mathrm{V}$-BLAST layer in the first time slot are given by

$$
\tilde{\mathrm{y}}_{i, 1}=\mathrm{R}_{i, i} \nu_{i, 1}+\sum_{j=i+1}^{M-2} \mathrm{R}_{i, j} \hat{v}_{j, 1}+\left(\mathrm{R}_{i, M-1} \tilde{s}_{M-1}+\mathrm{R}_{i, M} \tilde{s}_{M}\right)+\tilde{\mathbf{z}}_{i, 1}
$$

while the received sub-data streams of V-BLAST layer in the second time slot are given by 
Table 1 Comparison of number of complex arithmetic operations for ZF, MMSE, QR decomposition and LC-QR decomposition detection mechanisms

\begin{tabular}{|c|c|c|c|c|}
\hline Complex arithmetic operation & ZF with $\vec{H}$ & MMSE with $\vec{H}$ & QR decomposition with $\vec{H}$ & LC-QR with $\mathrm{H}$ \\
\hline Addition & $4(4 \boldsymbol{N}-1) \times(\boldsymbol{M}-1)^{2}-2(\boldsymbol{M}-1)$ & $4(4 \boldsymbol{N}-1) \times(\boldsymbol{M}-1)^{2}$ & $2(2 \boldsymbol{N}-1) \times(\boldsymbol{M}-1)+(\boldsymbol{M}-2) \times(2 \boldsymbol{M}-3)$ & $\boldsymbol{N}(3 \boldsymbol{M}-2)+(\boldsymbol{M}-3) \times(\boldsymbol{M}-1)$ \\
\hline Subtraction & - & - & $2 \boldsymbol{M}-3$ & $2(\boldsymbol{M}-2)+2 \boldsymbol{N}+1$ \\
\hline Multiplication & $4 \boldsymbol{N}(\boldsymbol{M}-1) \times(4 \boldsymbol{M}-3)$ & $4 \boldsymbol{N}(\boldsymbol{M}-1) \times(4 \boldsymbol{M}-3)$ & $(\boldsymbol{M}-1) \times(4 \boldsymbol{N}+2 \boldsymbol{M}-3)$ & $\boldsymbol{M}^{2}+3 \boldsymbol{M N}-\boldsymbol{M}+4$ \\
\hline Division & - & - & $2(\boldsymbol{M}-1)$ & $2(\boldsymbol{M}-2)$ \\
\hline Householder reflection & - & - & $8 \boldsymbol{N}(\boldsymbol{M}-1)^{2}-(8 / 3)(\boldsymbol{M}-1)^{3}$ & $N M^{2}-M^{3 / 3}$ \\
\hline Gaussian elimination & $8(\boldsymbol{M}-1)^{3}$ & $8(\boldsymbol{M}-1)^{3}$ & - & - \\
\hline Total & $\begin{array}{l}8(\boldsymbol{M}-1)^{3}+4(8 \boldsymbol{N}-1)(\boldsymbol{M}-1)^{2}+ \\
2(2 \boldsymbol{N}-1)(\boldsymbol{M}-1)\end{array}$ & $\begin{array}{l}8(\boldsymbol{M}-1)^{3}+4(8 \boldsymbol{N}-1)(\boldsymbol{M}-1)^{2}+ \\
4 \boldsymbol{N}(\boldsymbol{M}-1)\end{array}$ & $\begin{array}{l}8 \boldsymbol{N}(\boldsymbol{M}-1)^{2}-(8 / 3)(\boldsymbol{M}-1)^{3}+(2 \boldsymbol{M}-3)^{2}+ \\
2(\boldsymbol{M}-1)(4 \boldsymbol{N}+1)-1\end{array}$ & $\begin{array}{l}\boldsymbol{N} \boldsymbol{M}^{2}-\boldsymbol{M}^{3} / 3+(\boldsymbol{M}+2)(2 \boldsymbol{N}-1)+2(\boldsymbol{M}-2)(\boldsymbol{M}+2)+ \\
4 \boldsymbol{N}(\boldsymbol{M}-1)+10\end{array}$ \\
\hline
\end{tabular}


Table 2 Reduction of computational complexity for ZF, MMSE and QR decomposition compared to LC-QR decomposition detection mechanisms

\begin{tabular}{|c|c|c|c|c|c|c|c|c|}
\hline \multirow[t]{3}{*}{$\begin{array}{l}\text { Complex arithmetic } \\
\text { operation }\end{array}$} & \multicolumn{4}{|c|}{$\begin{array}{l}\text { Reduction of computational } \\
\text { complexity compared to ZF and } \\
\text { MMSE with } \vec{H}(\%)\end{array}$} & \multicolumn{4}{|c|}{$\begin{array}{l}\text { Reduction of computational } \\
\text { complexity compared to QR } \\
\text { decomposition with } \vec{H}(\%)\end{array}$} \\
\hline & \multicolumn{2}{|l|}{$M=4$} & \multicolumn{2}{|l|}{$M=5$} & \multicolumn{2}{|l|}{$M=4$} & \multicolumn{2}{|l|}{$M=5$} \\
\hline & $N=4$ & $N=5$ & $N=5$ & $N=6$ & $N=4$ & $N=5$ & $N=5$ & $N=6$ \\
\hline Addition & 93.2 & 92.2 & 94.0 & 94.1 & 17.3 & 17.2 & 21.5 & 21.1 \\
\hline Subtraction & - & - & - & - & - & - & - & - \\
\hline Multiplication & 89.7 & 90.3 & 92.7 & 93.0 & - & - & 8.3 & 8.1 \\
\hline Division & - & - & - & - & 33.3 & 33.3 & 25.0 & 25.0 \\
\hline Householder reflection & - & - & - & - & 80.6 & 79.9 & 82.1 & 81.8 \\
\hline Gaussian elimination & - & - & - & - & - & - & - & - \\
\hline Total & 87.9 & 87.7 & 90.9 & 90.7 & 51.5 & 53.0 & 59.3 & 60.5 \\
\hline
\end{tabular}

$$
\tilde{\mathrm{y}}_{i, 2}=\mathrm{R}_{i, i} v_{i, 2}^{*}+\sum_{j=i+1}^{M-2} \mathrm{R}_{i, j} \hat{v}_{j, 2}^{*}+\left(\mathrm{R}_{i, M-1}\left(-\tilde{s}_{M}^{*}\right)+\mathrm{R}_{i, M}\left(\tilde{s}_{M-1}^{*}\right)\right)+\tilde{\mathbf{z}}_{i, 2}
$$

where $i=1, \ldots, M-2$. Here, it is assumed that when the V-BLAST layer symbols are decoded, the previously decoded first estimated symbols of STBC layer have been cancelled properly. Hence, decoding of $\hat{v}_{i, 1}$ and $\hat{v}_{i, 2}$ become

$$
\begin{aligned}
& \hat{v}_{i, 1}=v_{i, 1}+\frac{\tilde{\mathrm{z}}_{i, 1}}{\mathrm{R}_{i, i}} \\
& \hat{v}_{i, 2}=\left(v_{i, 2}^{*}+\frac{\tilde{\mathbf{z}}_{i, 2}}{\mathrm{R}_{i, i}}\right)^{*}
\end{aligned}
$$

It can be observed from (25) and (26) that the SNR of $i$-th detected sub-data stream of V-BLAST layer over two time slots is determined by the diagonal element $\left|\mathrm{R}_{i, i}\right|^{2}$ of $\mathrm{R}$ where $i=1,2$. So, the SNR of $i$-th detected sub-data stream, denoted as $\rho_{i}$, becomes

$$
\rho_{i}=\frac{\rho\left|\mathrm{R}_{i, i}\right|^{2}}{M}
$$

Since the V-BLAST layer of V-BLAST/STBC scheme transmits four data symbols over two consecutive symbol transmission periods, the instantaneous system capacity of the V-BLAST layer of LC-QR decomposition, denoted as $C_{P_{-} V-B L A S T}$, is $[26]$

$$
C_{P_{-} V-B L A S T}=\frac{4 \log _{2}\left(1+\min _{i \in\{1, \ldots, M-2\}} \rho_{i}\right)}{2}=2 \log _{2}\left(1+\min _{k \in\{1, \ldots, M-2\}} \frac{\rho\left|R_{i, i}\right|^{2}}{4}\right)
$$


Therefore, the total capacity of V-BLAST/STBC Scheme with LC-QR decomposition, denoted as $C_{P_{-} L C-Q R}$, is the summation of $C_{P_{-} S T B C}$ and $C_{P_{-} V-B L A S T}$ as

$$
\begin{aligned}
C_{P_{-} L C-Q R}=C_{P_{-} S T B C}+C_{P_{-} V-B L A S T}= & \log _{2}\left(1+\frac{\rho}{4}\left(\left|\mathrm{R}_{M-1, M-1}\right|^{2}+\left|\mathrm{R}_{M-1, M}\right|^{2}+\left|\mathrm{R}_{M, M}\right|^{2}\right)\right) \\
& +2 \log _{2}\left(1+\min _{k \in\{1, \ldots, M-2\}} \frac{\rho\left|\mathrm{R}_{i, i}\right|^{2}}{4}\right)
\end{aligned}
$$

\section{Probability of error in V-BLAST/STBC scheme with LC-QR decomposition}

A MIMO communication system with $M$ transmit and $N$ receive antennas is considered in a Rayleigh frequency flat-fading channel. The received signal is given by

$$
\mathrm{y}=\mathrm{Hx}+\mathrm{z}
$$

where y is the $N \times 2$ received signal matrix and $\mathrm{H}$ is the $N \times M$ Rayleigh flat fading channel matrix. $\mathrm{z}$ is the AWGN $N \times 2$ complex matrix with unit variance $\sigma_{z}^{2}$ and zero mean. It is assumed that the entries of $\mathrm{H}$ are circularly symmetric, independent and identically distributed (i.i.d.) with zero-mean and unit variance $\sigma_{h}^{2}$. It is also assumed that the transmission matrix and with uniform power is independent. The covariance matrix of $\mathrm{x}$ is $E\left[\mathbf{x x}^{*}\right]=\sigma_{x}^{2} I$, where $E[\bullet]$ denotes the expected value and $(\bullet)^{*}$ is the conjugate transpose. The SNR, denoted as $\gamma$, is defined as below

$$
\gamma=\frac{\sigma_{x}^{2}}{\sigma_{z}^{2}}
$$

\section{Analytical model of V-BLAST layer}

Denote the $\mathrm{QR}$ decomposition of $\mathrm{H}$ as $\mathrm{H}=\mathrm{QR}$. The matrix $\mathrm{R}$ is upper triangular matrix with diagonal real number elements. The entries of $\mathrm{R}$ are independent of each other. The off diagonal elements $\mathrm{R}_{i}$, for $1 \leq i<j \leq M$, are zero-mean complex Gaussian with unit variance. The square of the $i$-th diagonal element of $\mathrm{R}$, denoted as $\mathrm{R}_{i i}^{2}$, is a chi-square distribution with the $2 k$ degrees of freedom, where $k$ is determined by the diversity gain of V-BLAST scheme. Therefore, the probability density function (PDF) of post-detection SNR of V-BLAST scheme with chi-square distribution and $2 k$ degrees of freedom, denoted as $f_{V}(x, 2 k)$, is given by [27]

$$
f_{V}(x, 2 k)=\frac{1}{\sigma^{2 k} 2^{k} \Gamma(k)} x^{k-1} e^{\left(-x / 2 \sigma^{2}\right)}
$$

where $\Gamma(k)$ is the gamma function given by

$$
\Gamma(k)=\int_{0}^{\infty} t^{k-1} e^{-t} d t, \quad k>0
$$

If $k$ is a positive integer, then $\Gamma(k)=(k-1)$ !. The received V-BLAST/STBC matrix is given by $\tilde{y}=\mathbf{R x}+\tilde{z}$ as in (13). Hence, the received sub-data streams of V-BLAST layer in the first and second time slot are given by

$$
\tilde{\mathrm{y}}_{i, 1}=\mathrm{R}_{i, i} \nu_{i, 1}+\sum_{j=i+1}^{M-2} \mathrm{R}_{i, j} \hat{v}_{j, 1}+\left(\mathrm{R}_{i, M-1} \tilde{s}_{M-1}+\mathrm{R}_{i, M} \tilde{s}_{M}\right)+\tilde{\mathbf{z}}_{i, 1}
$$




$$
\tilde{\mathrm{y}}_{i, 1}=\mathrm{R}_{i, i} \nu_{i, 1}+\sum_{j=i+1}^{M-2} \mathrm{R}_{i, j} \hat{v}_{j, 1}+\left(\mathrm{R}_{i, M-1} \tilde{s}_{M-1}+\mathrm{R}_{i, M} \tilde{s}_{M}\right)+\tilde{\mathbf{z}}_{i, 1}
$$

where $i=1, \ldots, M-2$. It is assumed that the propagation error effect is cancelled properly, then (42) and (43) are simplified to

$$
\begin{aligned}
& \tilde{y}_{i, 1}=\mathrm{R}_{i, i} v_{i, 1}+\tilde{\mathrm{z}}_{i, 1} \\
& \tilde{\mathrm{y}}_{i, 2}=\mathrm{R}_{i, i} v_{i, 2}^{*}+\tilde{\mathrm{z}}_{i, 2}
\end{aligned}
$$

The instantaneous post-detection SNR is determined by the $\mathrm{R}_{i i}^{2}$. It is shown in [28] that the diversity gain of $i$-th detected sub-data stream layer of V-BLAST scheme is $(N-i+1)$. Thus, $\mathrm{R}_{i i}^{2}$ is a chi-square distribution with the degree of freedom 2 $(N-i+1)$, which is denoted as $X_{2(N-i+1)}^{2}$. It can be observed that the when the $i$ is larger, the diversity gain of the $i$-th layer becomes smaller. Consequently, the largest or $M$-th layer limits the overall performance of V-BLAST scheme at high SNR. Therefore, the overall diversity gain of V-BLAST scheme is $N-M+1$ [29].

After that, $M_{V}$ is defined as the number of transmit antenna of V-BLAST layer in V-BLAST/STBC scheme. Then, the effective diversity gain of V-BLAST layer in V-BLAST/STBC scheme becomes $N-M_{V}+1$. Thus, substituting $k=N-M_{V}+1$ into (41), the PDF of instantaneous post-detection SNR of V-BLAST layer in V-BLAST/ STBC scheme with chi-square distribution and $2\left(N-M_{V}+1\right)$ degrees of freedom, denoted as $f_{V S}^{V}\left(x, 2\left(N-M_{V}+1\right)\right)$, is given by

$$
f_{V S}^{V}\left(x, 2\left(N-M_{V}+1\right)\right)=\frac{1}{\sigma^{2\left(N-M_{V}+1\right)} 2^{\left(N-M_{V}+1\right)} \Gamma\left(N-M_{V}+1\right)} x^{N-M_{V}} e^{\left(-x / 2 \sigma^{2}\right)}
$$

It is shown in [30] that BER of M-ary quadrature amplitude modulation (M-QAM) over AWGN noise is given by

$$
P_{e, \mathrm{M} \text {-QAM }}(\gamma)=\frac{4}{\log _{2} \mathrm{M}}\left(1-\frac{1}{\sqrt{\mathrm{M}}}\right) \times \sum_{i=1}^{\sqrt{\mathrm{M}} / 2} Q\left((2 i-1) \sqrt{\frac{3 \gamma \log _{2} \mathrm{M}}{(\mathrm{M}-1)}}\right)
$$

where $Q(\bullet)$ is Gaussian $Q$-Function given by

$$
Q(x)=\frac{1}{2}\left(1-\operatorname{erf}\left(\frac{x}{\sqrt{2}}\right)\right)
$$

and the error function $\operatorname{erf}(\bullet)$ is defined as

$$
\operatorname{erf}(x)=\frac{2}{\sqrt{\pi}} \int_{0}^{x} e^{-t^{2}} d t
$$

It is shown in [31] that the average error probability can be obtained by averaging the conditional error probability of modulation scheme over the PDF of instantaneous post-detection SNR of MIMO scheme. Therefore, the BER performance of V-BLAST 
layer in V-BLAST/STBC scheme with M-QAM modulation, $M_{V}$ and $N$, denoted as $P_{e, \mathrm{M}-\mathrm{QAM}, V S}^{V}\left(M_{V}, N, \gamma\right)$, can be computed by integrating (46) and (47) to become

$$
\begin{aligned}
P_{e, \mathrm{M}-\mathrm{QAM}, V S}^{V}\left(M_{V}, N, \gamma\right)= & \int_{0}^{\infty} P_{e, \mathrm{M}-\mathrm{QAM}}(\gamma) f_{V S}^{V}\left(x, 2\left(N-M_{V}+1\right)\right) d x \\
= & \int_{0}^{\infty} \frac{4}{\log _{2} \mathrm{M}}\left(1-\frac{1}{\sqrt{\mathrm{M}}}\right) \times \sum_{i=1}^{\sqrt{\mathrm{M}} / 2} \mathrm{Q}\left((2 i-1) \sqrt{\frac{3 \log _{2} \mathrm{M} \gamma x}{(\mathrm{M}-1)}}\right) \\
& \times \frac{1}{\sigma^{2\left(N-M_{V}+1\right)} 2^{\left(N-M_{V}+1\right)} \Gamma\left(N-M_{V}+1\right)} x^{N-M_{V}} e^{\left(-x / 2 \sigma^{2}\right)} d x
\end{aligned}
$$

\section{Analytical model of STBC layer}

From (18), interference cancellation is performed by subtracting the data symbols of V-BLAST layer. Before interference cancellation, the received $i$-th sub-data stream in $l$-th time slot is given by

$$
\underbrace{\mathrm{y}_{i, l}=h_{i, M-1} s_{M-1}+h_{i, M} s_{M}}_{\begin{array}{c}
\text { STBClayer } \\
\text { symbols }
\end{array}}+\underbrace{\sum_{j=i}^{M-2} h_{i, j} v_{j, l}+\mathrm{z}_{i, l}}_{\begin{array}{c}
\text { Interference } \\
\text { AWGN noise }
\end{array}}
$$

It can be seen from (51) that the received $i$-th sub-data stream is composed of STBC layer symbols, AWGN noise and the potential propagation error from V-BLAST layer. The equivalent noise is the combination of the last two parts.

In the ideal case where there is no propagation error from V-BLAST layer, the BER analysis of STBC layer is the same as BER analysis of the Alamouti's STBC scheme with two transmit and $N$ receive antennas. The instantaneous post-detection SNR of Alamouti's STBC scheme with two transmit and $N$ receive antennas, denoted as $\gamma_{S}$, is given by

$$
\gamma_{S}=\gamma \sum_{i=1}^{N}\left(\left|h_{i, 1}\right|^{2}+\left|h_{i, 2}\right|^{2}\right)
$$

Since $h_{i, 1}$ and $h_{i, 2}$ are circularly symmetric, i.i.d. Gaussian random variables with zero-mean and unit variance, the $\gamma_{S}$ has a chi-square distribution with $2 \times 2 \times N$ degrees of freedom.

The diversity gain of O-STBC scheme is given by $M \times N$ [32]. So, the PDF of instantaneous post-detection SNR of O-STBC scheme with chi-square distribution and $2 \times$ $M \times N$ degrees of freedom, denoted as $f_{S}(x, 2 M N)$, is given by

$$
f_{S}(x, 2 M N)=\frac{1}{\sigma^{2 M N} 2^{M N} \Gamma(M N)} x^{M N-1} e^{\left(-x / 2 \sigma^{2}\right)}
$$

The BER of O-STBC scheme with $M$ transmit and $N$ receive antennas and M-QAM modulation, denoted as $P_{e, \mathrm{M} \text {-QAM }}^{S}(M, N, \gamma)$, can be calculated by integrating (47) and (53) as follows 


$$
\begin{aligned}
P_{e, \mathrm{M}-\mathrm{QAM}}^{S}(M, N, \gamma)= & \int_{0}^{\infty} P_{e, \mathrm{M}-\mathrm{QAM}}(\gamma) f_{S}(x, 2 M N) d x \\
= & \int_{0}^{\infty} \frac{4}{\log _{2} \mathrm{M}}\left(1-\frac{1}{\sqrt{\mathrm{M}}}\right) \times \sum_{i=1}^{\sqrt{\mathrm{M}} / 2} \mathrm{Q}\left((2 i-1) \sqrt{\frac{3 \log _{2} \mathrm{M} \gamma x}{(\mathrm{M}-1)}}\right) \\
& \times \frac{1}{\sigma^{2 M N} 2^{M N} \Gamma(M N)} x^{M N-1} e^{\left(-x / 2 \sigma^{2}\right)} d x
\end{aligned}
$$

From (19) and (20), the STBC layer of V-BLAST/STBC scheme is decoded with STBC $2 \times N$ decoder after the V-BLAST layer is decoded. Therefore, the BER performance of V-BLAST layer dominates the BER performance of STBC layer. The statistical properties of BER performance of STBC layer are said to be conditional.

$M_{S}$ is defined as the number of transmit antenna of Alamouti's STBC layer in V-BLAST/STBC scheme, where $M_{S}=2$. On the other hand, an event with $k$ symbol errors detection in V-BLAST layer, denoted as $A_{k}$, is defined where $k \leq 2 M_{V}$. Then, $\left(P_{e, \mathrm{M} \text {-QAM }}^{S}\left(M_{S}, N, \gamma\right) \mid A_{k}\right)$ is defined as the $P_{e, \mathrm{M} \text {-QAM }}^{S}\left(M_{S}, N, \gamma\right)$ after the event $A_{k}$ happens. On the other hand, the symbol error rate (SER) of V-BLAST layer with $M_{V}$ transmit, $N$ receive antennas and M-QAM modulation over AWGN noise, denoted as $P_{S E R, \mathrm{M}-\mathrm{QAM}}^{V}\left(M_{V}, N, \gamma\right)$, can be expressed as [33]

$$
P_{S E R, \mathrm{M}-\mathrm{QAM}}^{V}\left(M_{V}, N, \gamma\right)=2\left(1-\frac{1}{\sqrt{\mathrm{M}}}\right) \operatorname{erfc}\left(\sqrt{\frac{3 \gamma}{2(\mathrm{M}-1)}}\right)
$$

where $\operatorname{erfc}(x)=1-\operatorname{erf}(x)$.

Since there is no symbol errors detection in V-BLAST layer, the $A_{k}$ becomes $A_{0}$. Hence, the BER performance of STBC layer in V-BLAST/STBC scheme in ideal case is the same as the BER performance of Alamouti's STBC scheme. Therefore, the BER performance of STBC layer in V-BLAST/STBC scheme in the ideal case with M-QAM modulation, $M_{S}$ and $N$, denoted as $P_{e, \mathrm{M} \text {-QAM, } V S}^{\text {ideal }}\left(M_{S}, N, \gamma\right)$, can be computed as

$$
P_{e, \mathrm{M}-\mathrm{QAM}, V S}^{\text {ideal }}\left(M_{S}, N, \gamma\right)=\left(P_{e, \mathrm{M}-\mathrm{QAM}}^{S}\left(M_{S}, N, \gamma\right) \mid A_{0}\right) \times\left(1-\left(P_{S E R, \mathrm{M}-\mathrm{QAM}}^{V}\left(M_{V}, N, \gamma\right)\right)\right)
$$

where $\left(P_{e, \mathrm{M}-\mathrm{QAM}}^{S}\left(M_{S}, N, \gamma\right) \mid A_{0}\right)=P_{e, \mathrm{M}-\mathrm{QAM}}^{S}\left(M_{S}, N, \gamma\right)$.

In the non-ideal case, there is a propagation error from V-BLAST layer as more than or equal to one symbols of V-BLAST layer are decoded wrongly. The $\sigma_{V}^{2}$ and $\sigma_{S}^{2}$ are defined as unit variance of V-BLAST and STBC layer respectively, where $\sigma_{V}^{2}=\sigma_{S}^{2}=\sigma_{x}^{2}$. From (51), the combination of interference and AWGN noise with $k$ V-BLAST symbol errors detection can be written as $\sigma_{z}^{2}+k \sigma_{V}^{2}$. Then, the effective SNR for STBC layer with $k$ V-BLAST symbol errors detection, denoted as $\tilde{\gamma}_{(k)}$, can be written as

$$
\tilde{\gamma}_{(k)}=\frac{\sigma_{S}^{2}}{\sigma_{z}^{2}+k \sigma_{V}^{2}}
$$

where $\sigma_{z}^{2} \neq 0$. Dividing (57) by $\sigma_{z}^{2}$, will get

$$
\tilde{\gamma}_{(k)}=\frac{\gamma_{S}}{1+k \gamma_{V}}
$$


where $\gamma_{V}=\gamma_{S}=\gamma$. Figure 3 shows the block diagram of analytical model of STBC layer in V-BLAST/STBC scheme.

The BER performance of STBC layer in V-BLAST/STBC scheme in non-ideal case with $k$-th detection V-BLAST symbol errors, M-QAM modulation, $M_{S}$ and $N$, denoted as $P_{e, \mathrm{M} \text {-QAM, } \text {-QRS }}^{\text {non-eal }}\left(M_{S}, N, \tilde{\gamma}_{(k)}\right)$, can be computed as

$$
\begin{aligned}
P_{e, \mathrm{M}-\mathrm{QAM}, V S}^{\text {non-ideal }}\left(M_{S}, N, \tilde{\gamma}_{(k)}\right)= & \left(P_{e, \mathrm{M} \text {-QAM }}^{S}\left(M_{S}, N, \tilde{\gamma}_{(k)}\right) \mid A_{k}\right) \times\left(P_{S E R, \mathrm{M}-\mathrm{QAM}}^{V}\left(M_{V}, N, \gamma\right)\right)^{k} \\
= & \sum_{k=1}^{2 M_{V}} P_{e, \mathrm{M}-\mathrm{QAM}}^{S}\left(M_{S}, N, \tilde{\gamma}_{(k)}\right) \times\left(\begin{array}{c}
2 M_{V} \\
k
\end{array}\right) \times\left(P_{S E R, \mathrm{M}-\mathrm{QAM}}^{V}\left(M_{V}, N, \gamma\right)\right)^{k} \\
& \times\left(1-P_{S E R, \mathrm{M}-\mathrm{QAM}}^{V}\left(M_{V}, N, \gamma\right)\right)^{2 M_{V}-k}
\end{aligned}
$$

where

$$
\left(\begin{array}{c}
2 M_{V} \\
k
\end{array}\right)=\frac{\left(2 M_{V}\right) !}{k !\left(2 M_{V}-k\right) !}
$$

which is the number of $k$ permutations of an $2 M_{V}$-element set. Meanwhile, $\left(P_{S E R, \mathrm{M}-\mathrm{QAM}}^{V}\left(M_{V}, N, \gamma\right)\right)^{k} \times\left(1-P_{S E R, \mathrm{M}-\mathrm{QAM}}^{V}\left(M_{V}, N, \gamma\right)\right)^{2 M_{V}-k}$ denotes the probability of error of V-BLAST layer with event $A_{k}$.

It is known that $P_{S E R, \mathrm{M}-\mathrm{QAM}}^{V}\left(M_{V}, N, \gamma\right) \neq 0$ for all SNR when $\sigma_{z}^{2} \neq 0$. As long as $P_{S E R, \mathrm{M}-\mathrm{QAM}}^{V}\left(M_{V}, N, \gamma\right) \neq 0, k$ exists for $1, \ldots, 2 M_{V}$. So, it can deduced that $\sigma_{z}^{2}$ is uncorrelated with $k$ when $\sigma_{z}^{2} \neq 0$. Finally, the BER performance of STBC layer in V-BLAST/ STBC scheme with M-QAM modulation, $M_{V}$ and $N$, denoted as $P_{e, \mathrm{M}-\mathrm{QAM}, V S}^{S}\left(M_{V}, N, \gamma\right)$, can be computed as

$$
P_{e, \mathrm{M} \text {-QAM }, V S}^{S}\left(M_{V}, N, \gamma\right)=P_{e, \mathrm{M}-\mathrm{QAM}, V S}^{\text {ideal }}\left(M_{S}, N, \gamma\right)+P_{e, \mathrm{M}-\mathrm{QAAM}, V S}^{\text {non-ideal }}\left(M_{S}, N, \tilde{\gamma}_{(k)}\right)
$$

\section{Analytical model of V-BLAST/STBC scheme}

By combining (50) and (61), the final overall BER performance $P_{e, \mathrm{M} \text {-QAM, } V S}^{O}(M, N)$ for V-BLAST/STBC scheme with M-QAM modulation, $M$ transmit and $N$ receive antennas can be computed as

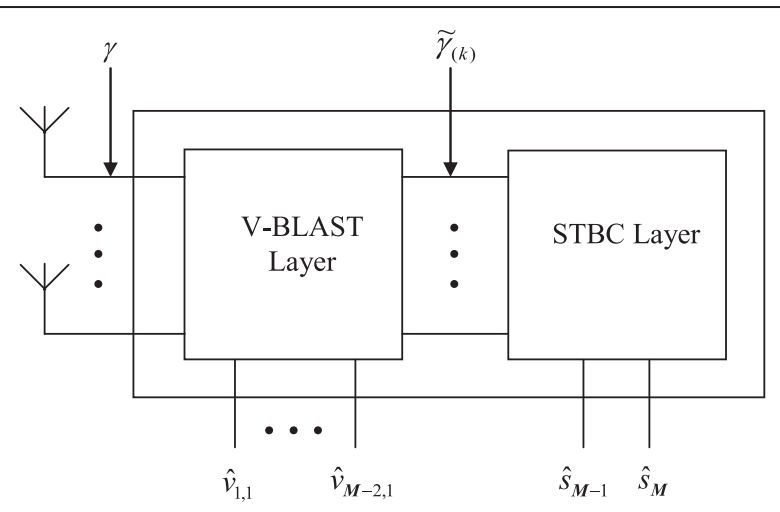

Figure 3 Block diagram of analytical model of STBC layer in V-BLAST/STBC scheme. 


$$
P_{e, \mathrm{M}-\mathrm{QAM}, V S}^{O}(M, N)=\frac{\mathrm{m} \times P_{e, \mathrm{M}-\mathrm{QAM}, V S}^{V}\left(M_{V}, N, \gamma\right)+\mathrm{n} \times P_{e, \mathrm{M}-\mathrm{QAM}, V S}^{S}\left(M_{V}, N, \gamma\right)}{\mathrm{m}+\mathrm{n}}
$$

where $M_{S}=2$ and $M_{S}+M_{V}=M$. The $\mathrm{m}$ and $\mathrm{n}$ are the symbol transmission rates of $\mathrm{V}$-BLAST and STBC layer respectively.

\section{Performance evaluation of LC-QR decomposition in V-BLAST/STBC scheme}

The simulations have been performed on the MIMO system using MATLAB to evaluate the performance of the V-BLAST/STBC scheme with ZF, MMSE and QR decomposition with $\vec{H}$ and LC-QR decomposition with $\mathrm{H}$. The 4-ary quadrature amplitude modulation (4-QAM) constellation is used in these simulations with Rayleigh flat-fading channel as well as Naftali channel. The entries of Rayleigh flat-fading channel matrix are circularly symmetric, i.i.d. Gaussian random variables with zero-mean and unit variance. The symbol rate of ZF V-BLAST $4 \times 4$, O-STBC $4 \times 4$ and V-BLAST/STBC $4 \times 4$ is shown in Table 3.

Figure 4 depicts the $10 \%$ and $1 \%$ outage capacity for MIMO (theoretical limit), ZF V-BLAST, O-STBC and V-BLAST/STBC with LC-QR decomposition in $4 \times 4$ system. The spectral efficiency of ZF V-BLAST changes a lot with different outage probability. For instance, ZF V-BLAST requires $8 \mathrm{~dB}$ to maintain the capacity of $15 \mathrm{bps} / \mathrm{Hz}$ when it proceeds from $10 \%$ to $1 \%$ outage probability. This is caused by lack of diversity of ZF V-BLAST. In contrast, the V-BLAST/STBC with LC-QR decomposition just requires $3 \mathrm{~dB}$ to maintain at the capacity of $15 \mathrm{bps} / \mathrm{Hz}$. Last but not least, the O-STBC is the most stable one, as the curve of $10 \%$ outage capacity is very close to the curve of $1 \%$ outage capacity. Besides, it can be observed that the capacity of ZF V-BLAST with $10 \%$ outage probability is the highest among the considered schemes for SNR higher than $37 \mathrm{~dB}$. Meanwhile, the capacity of VBLAST/STBC with $10 \%$ outage probability is close to MIMO capacity at low SNR.

Figure 5 shows the BER performance comparison of ZF V-BLAST $4 \times 4$, O-STBC $4 \times 4$ with rate $3 / 4$ and V-BLAST/STBC $4 \times 4$ with LC-QR decomposition in Rayleigh flat-fading channel environment, which is constant across four consecutive symbol transmission periods. It could be seen that the BER performance of O-STBC $4 \times 4$ with symbol rate $3 / 4$ is the best among the considered schemes as it is a pure spatial diversity scheme with full diversity gain. Moreover, O-STBC does not suffer from inter-symbol interference (ISI) as the transmitted symbols are orthogonal to one another. In contrast, ZF V-BLAST $4 \times 4$ with symbol rate four is the worst among the schemes because it is a pure spatial multiplexing scheme which suffers from poor diversity gain. Besides, interference between transmitted symbols in ZF V-BLAST scheme greatly reduces the BER performance. It could be seen that the V-BLAST/STBC $4 \times 4$ with symbol rate three shows a compromise of BER performance with respect to pure spatial multiplexing or diversity scheme.

Table 3 Symbol rate of various $4 \times 4$ MIMO schemes

\begin{tabular}{ll}
\hline MIMO scheme & Symbol rate (symbol/symbol transmission period) \\
\hline ZF V-BLAST $4 \times 4$ & 4 \\
O-STBC $4 \times 4$ & $3 / 4$ \\
V-BLAST/STBC $4 \times 4$ & 3 \\
\hline
\end{tabular}




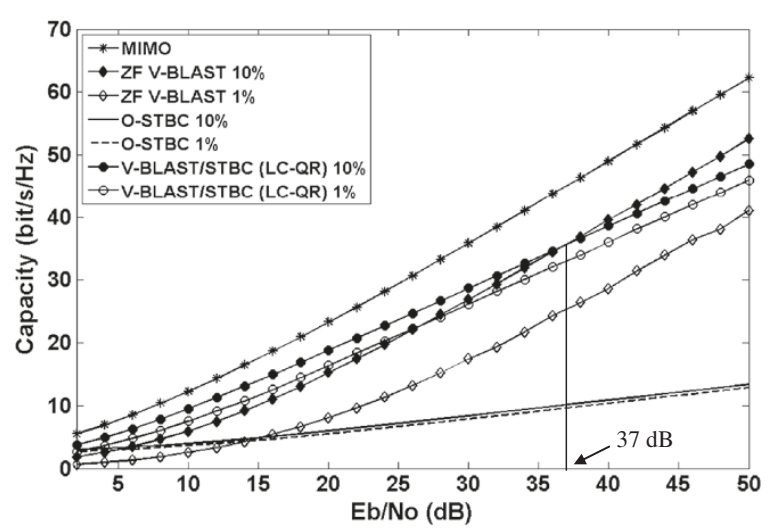

Figure 4 The $10 \%$ and $1 \%$ outage capacity comparison for various schemes $4 \times 4$.

It can be concluded that Figures 4 and 5 present the tradeoffs among ZF V-BLAST, $\mathrm{O}-\mathrm{STBC}$ and V-BLAST/STBC with LC-QR decomposition in $4 \times 4$ MIMO system. The O-STBC achieves the best BER performance, but the system capacity is the lowest among the considered schemes. Meanwhile, the system capacity of ZF V-BLAST with $10 \%$ outage capacity is the highest for SNR above $37 \mathrm{~dB}$, but the BER performance is the worst among the considered schemes. On the other hand, the system capacity of V-BLAST/STBC with LC-QR decomposition is close to MIMO and better than ZF V-BLAST for SNR below $37 \mathrm{~dB}$. Moreover, the BER performance of V-BLAST/ STBC with LC-QR decomposition is significantly better than ZF V-BLAST as V-BLAST/ STBC achieves spatial multiplexing and diversity gain simultaneously.

Figure 6 shows the BER performance of various mechanisms in V-BLAST/STBC scheme with Rayleigh flat-fading channel model, which is constant across two consecutive symbol transmission periods. The LC-QR decomposition $3 \times 2$ with $\mathrm{H}$ outperforms ZF $3 \times 2$ and MMSE $3 \times 2$ with $\vec{H}$ by more than $2 \mathrm{~dB}$ gain at BER of $10^{-3}$. At the same time, the LC-QR decomposition $3 \times 2$ with $\mathrm{H}$ outperforms $\mathrm{QR} 3 \times 2$ with $\vec{H}$ by approximately $1.5 \mathrm{~dB}$ gain at BER of $10^{-3}$. This is because the estimated candidate of STBC layer, which is more robust than V-BLAST layer, is decoded first. After decoding the

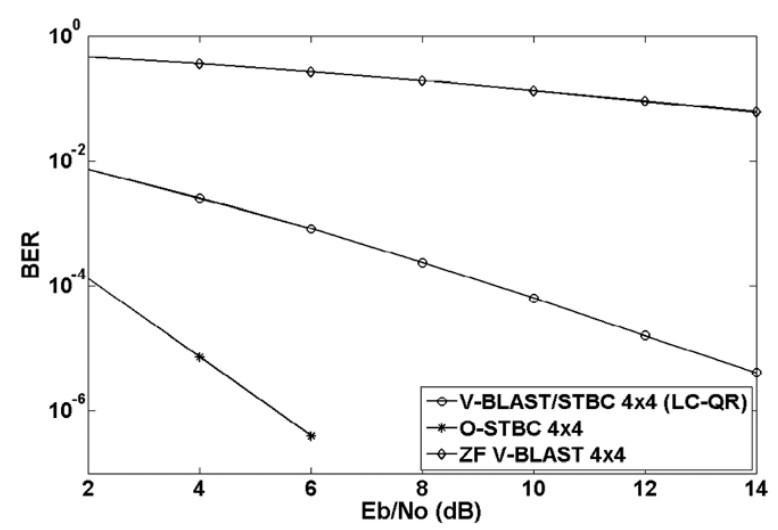

Figure 5 BER performance of various schemes $4 \times 4$. 


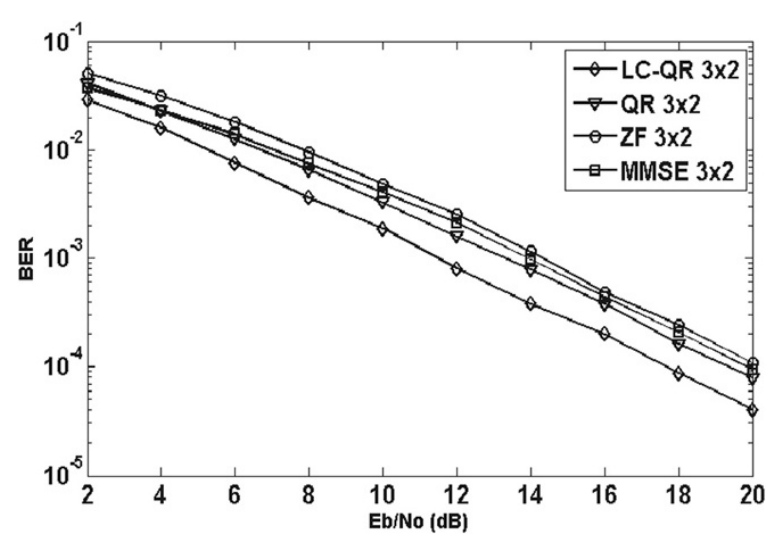

Figure 6 BER performance of various mechanisms in V-BLAST/STBC scheme with Rayleigh flat-fading channel model.

estimated candidate of STBC layer, interference cancellation activity is performed to produce a new modified received signal matrix $s$ with less interference. For ZF and MMSE mechanisms, there is no interference cancellation activity.

It is clear that V-BLAST layer performance dominates over final decisions of STBC layer in (25) and (26). With increasing SNR, the probability of error in decoding the V-BLAST layer is reduced, the probability of correct decoding is increased at the STBC layer. As the V-BLAST layer transmits four data symbols while STBC layer transmits two data symbols over two consecutive symbol transmission periods, thus a better BER performance of V-BLAST layer with LC-QR decomposition leads to overall V-BLAST/STBC system performance improvement.

Figure 7 illustrates the BER performance comparison of various mechanisms in V-BLAST/STBC scheme with a Naftali channel model under different maximum delay spread environment. The LC-QR decomposition $4 \times 4$ outperforms ZF $4 \times 4$ and MMSE $4 \times 4$ by approximately $2 \mathrm{~dB}$ gain at BER of $10^{-3}$ for both indoor (200 ns) and outdoor $(1.6 \mu \mathrm{s})$ environment. At the same time, the LC-QR decomposition $4 \times 4$ outperforms QR decomposition $4 \times 4$ by approximately $1 \mathrm{~dB}$ gain at BER of $10^{-3}$ for both indoor

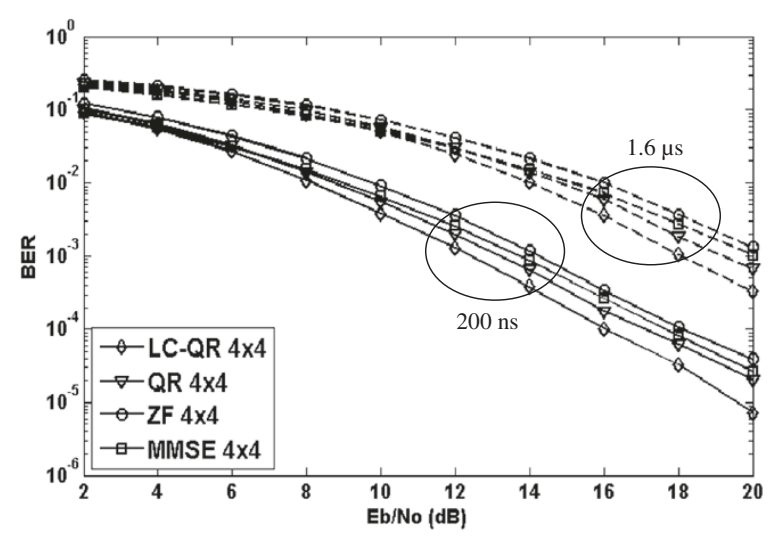

Figure 7 BER performance of various mechanisms in V-BLAST/STBC scheme under different maximum delay spread environment. 
and outdoor environment. In order to maintain the BER of $10^{-3}$, the LC-QR decomposition with a maximum delay spread $=1.6 \mu$ s requires $6 \mathrm{~dB}$ gain compared to indoor environment with a maximum delay spread $=200 \mathrm{~ns}$.

Figure 8 depicts BER performance of various mechanisms in V-BLAST/STBC scheme under different maximum delay spread environment at $16 \mathrm{~dB}$. From Figures 7 and 8 , it can be observed that performance BER of all considered mechanism degrades when the maximum delay spread is increasing because the delayed signal from multi-path overlaps the direct signal for the next symbol, thus ISI occurs. Moreover, it is well known that the delay spread causes frequency selective fading in the channel, which acts like a finite impulse response (FIR) filter. If the delay spread is comparable or larger than the symbol duration, the frequency-selective channel will distort the signal and not all frequency components fade simultaneously. As a result, the orthogonality of V-BLAST/ STBC data symbols could not be maintained, thus the BER performance declines.

Figure 9 plots the BER performance of LC-QR decomposition with different standard deviations of channel estimation error $\sigma_{\mathrm{E}}$ and delay spread $=200 \mathrm{~ns}$. On the other hand, Figure 10 shows the BER performance of various mechanisms in V-BLAST/STBC scheme with channel estimation error and delay spread $=200 \mathrm{~ns}$ at $16 \mathrm{~dB}$. It can be observed that the increase of standard deviation of channel estimation error decreases the BER of all considered mechanisms as channel estimation error is considered as an additional noise in the receiver. Consequently, irreducible error floor occurs when SNR increases in Figure 9. According to Figure 10, it can be deduced that all the considered mechanisms suffer from the same amount of noise variance from channel estimation error, because the equivalent channel matrix for ZF, MMSE and QR decomposition does not reduce the effect of channel estimation error.

Figure 11 illustrates the percentage of computational complexity reduction of the LC-QR decomposition with $\mathrm{H}$ compared to ZF, MMSE and QR decomposition with $\vec{H}$ for $N \geq M$. It can be observed that the LC-QR decomposition reduces the arithmetic operation complexity by at least $80 \%$ compared to ZF and MMSE with $\vec{H}$ as well as $35 \%$ compared to QR decomposition with $\vec{H}$. Thus, the LC-QR decomposition shows significant computational complexity improvement. The main reason is that the LCQR decomposition utilizes channel matrix $\mathrm{H}$ with smaller dimension instead of an

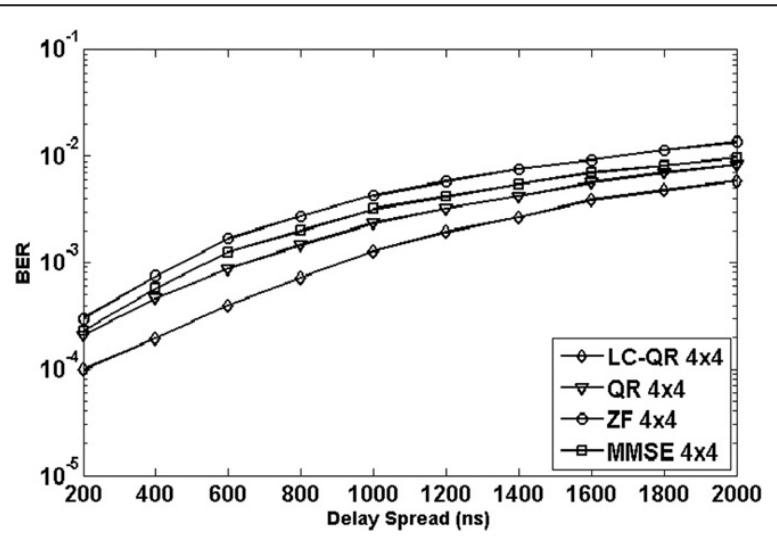

Figure 8 BER performance of various mechanisms in V-BLAST/STBC scheme under different maximum delay spread environment at $16 \mathrm{~dB}$. 


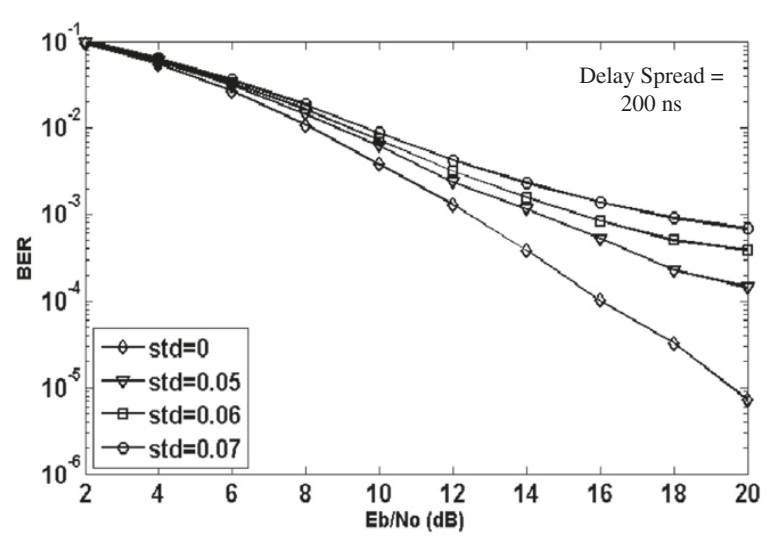

Figure 9 BER performance of LC-QR $4 \times 4$ with different standard deviations of channel estimation error $\sigma_{\mathrm{E}}$ and delay spread $=200 \mathrm{~ns}$.

equivalent channel matrix $\vec{H}$. It also can be seen that the percentage of computational complexity reduction of the LC-QR decomposition greatly increases when $M$ is increasing and $N$ is constant. This is due to the calculation of Gaussian elimination matrix inversion of $\vec{H}^{H} \vec{H}$ and $\left(\vec{H}^{H} \vec{H}+\sigma^{2} I_{2(M-1)}\right)$, which requires $8(M-1)^{3}$ complex arithmetic operation, for ZF and MMSE with $\vec{H}$ respectively. Note that the calculation of Gaussian elimination matrix inversion of $\vec{H}^{H} \vec{H}$ and $\left(\vec{H}^{H} \vec{H}+\sigma^{2} I_{2(M-1)}\right)$ does not need the information of $N$ and it increases significantly when $M$ becomes bigger. On the other hand, the calculation of QR decomposition with Householder reflection for $\vec{H}$ is $8 N(M-1)^{2}-(8 / 3)(M-1)^{3}$. Since $N$ is equal to or greater than $M$, the arithmetic operation of $8 N(M-1)^{2}-(8 / 3)(M-1)^{3}$ becomes significant when $M$ is increasing. The performance evaluations from these figures show that by using the proposed LC-QR decomposition detection mechanism in V-BLAST/STBC MIMO scheme the system performance is not only significantly improved but the computational complexity of the overall system is also significantly reduced. Hence, the computational cost and

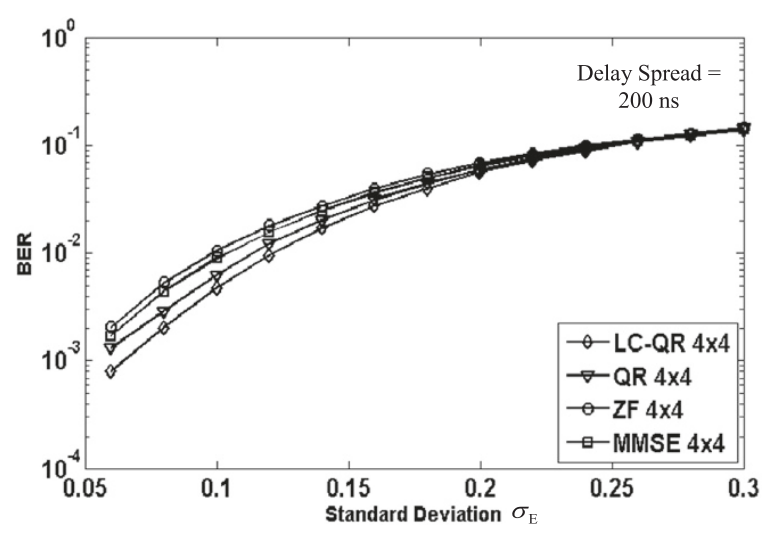

Figure 10 BER performance of various mechanisms in V-BLAST/STBC scheme $4 \times 4$ with different standard deviations of channel estimation error $\sigma_{\mathrm{E}}$ and delay spread $=200 \mathrm{~ms}$ at $16 \mathrm{~dB}$. 


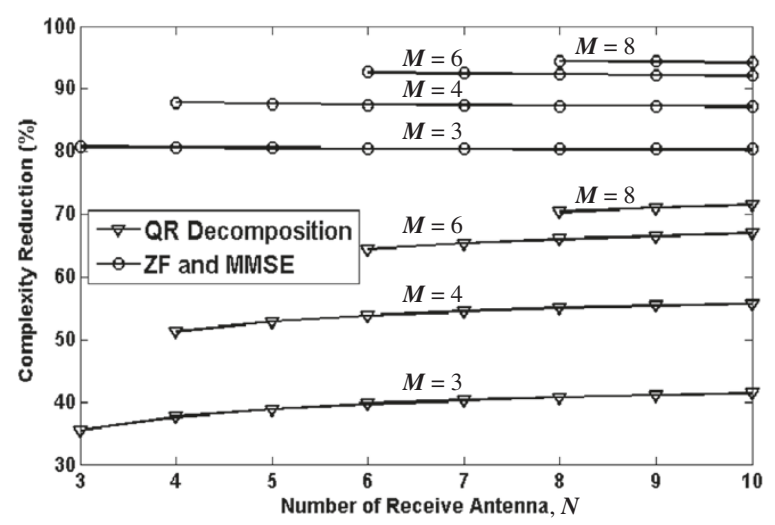

Figure 11 Percentage of computational complexity reduction of LC-QR decomposition compared to $Z F$, MMSE and QR decomposition with different $M$ and $N$ for $N \geq M$.

power consumption will be reduced for the next generation MIMO mobile computing devices can be reduced significantly from the reduction of computational complexity.

\section{Conclusions}

In this paper, it is illustrated that V-BLAST/STBC scheme, which achieves spatial multiplexing and diversity gains simultaneously, increases system capacity and maintains reliable BER performance to accommodate the ever growing demand for real time system with tolerably lower QoS. It is also shown that the system capacity of LC-QR decomposition V-BLAST/STBC scheme is close to the ideal MIMO system and better than ZF V-BLAST for SNR below $37 \mathrm{~dB}$. Moreover, the BER performance of LC-QR decomposition V-BLAST/STBC is significantly better than ZF-VBLAST. The LC-QR decomposition mechanism has also significantly reduced the arithmetic operation complexity and remains a satisfactory BER performance compared to ZF, MMSE and QR decomposition mechanisms. The reduction of computational complexity in V-BLAST/ STBC MIMO scheme will see a significant reduction in computational cost and power consumption for next generation MIMO mobile computing devices.

Competing interests

The authors declare that they have no competing interests.

Authors' contributions

JHC and CKN carried out the conceptualization, background study, simulated the concept, jointly drafted the manuscript and reviewed the manuscript. NKN and BMA carried out the conceptualization, jointly drafted the manuscript and reviewed the manuscript. All authors read and approved the final manuscript.

\section{Author details}

${ }^{1}$ Department of Computer Science and Mathematics, Faculty of Applied Sciences and Computing, Tunku Abdu Rahman University College, Jalan Genting Kelang, Setapak 53300 Kuala Lumpur, Malaysia. ${ }^{2}$ Institute of Gerontology, University Putra Malaysia, UPM Serdang 43400 Selangor, Malaysia. ${ }^{3}$ Department of Computer and Communication Systems Engineering, Faculty of Engineering, University Putra Malaysia, UPM Serdang 43400 Selangor, Malaysia.

Received: 12 January 2013 Accepted: 28 January 2014

Published online: 18 June 2014

\section{References}

1. Foschini GJ, Gans MJ (1998) On Limits of Wireless Communications in A Fading Environment When Using Multiple Antennas. Wirel Pers Commun 6:311-335

2. Mohamad R, Salleh WMHWM, Anas NM (2012) Multiband OFDM and OFDM Simulation Software using MATLAB ${ }^{\circledR}$ Graphical User Interface. J Convergence 3(1):1-4

3. Shbat MS, Tuzlukov V (2011) Dynamic Frequency Reuse Factor Choosing Method for Self Organizing LTE Networks. J Convergence 2(2):13-18 
4. Telatar E (1999) Capacity of Multi-Antenna Gaussian Channels. Eur Trans Telecommun 10(6):585-595

5. Golden GD, Foschini CJ, Valenzuela AR, Wolniansky PW (1999) Detection Mechanism and Initial Laboratory Results Using V-BLAST Space-Time Communication Architecture. Electron Lett 35(1):14-16

6. Tarokh V, Seshadri N, Calderbank AR (1998) Space-Time Codes for High Data Rate Wireless Communications: Performance Criterion and Code Construction. IEEE Trans Inf Theory 44(2):744-765

7. Alamouti SM (1998) A Simple Transmit Diversity Technique for Wireless Communications. IEEE J Sel Areas Commun 16(8):1451-1458

8. Tarokh V, Jafarkhani H, Calderbank AR (1999) Space-Time Block Codes from Orthogonal Designs. IEEE Trans Inf Theory 45(5):1456-1467

9. Zheng L, Tse DNC (2003) Diversity and Multiplexing: A Fundamental Tradeoff in Multiple Antenna Channels. IEEE Trans Inf Theory 49(5):1073-1096

10. Jafarkhani H (2005) Space Time Coding: Theory and Practice. Cambridge University Press, The Pitt Building, Trumpington Street, Cambridge, CB2 1RP United Kingdom

11. Wang H, Xia XG (2003) Upper Bounds of Rates of Complex Orthogonal Space-Time Block Codes. IEEE Trans Inf Theory 49(10):2788-2796

12. Luo H, Shyu ML (2011) Quality of Service Provision in Mobile Multimedia - A Survey. Human-centric Computing Information Sci 1(5):1-15

13. Bhattacharya A, Wu W, Yang Z (2012) Quality of Experience Evaluation of Voice Communication: An Affect-Based Approach. Human-centric Computing and Information Sci 2(7):1-18

14. Mao T, Motani M (2005) STBC-VBLAST for MIMO Wireless Communication Systems. Proc IEEE ICC (ICC 2005) 4:2266-2270

15. Meng C, Tuqan J (2007) Precoded STBC-VBLAST for MIMO Wireless Communication Systems. Proc IEEE Int Conf Acoust Spee (ICASSP 2007) 3:337-340

16. Thompson JS, Tan HS, Sun Y (2004) Investigation of Hybrid MIMO Techniques. In: Proceedings of 5th IEE International Conference on 3G Mobile Communication Technologies (3G 2004) 1-5

17. Longoria-Gandara O, Sanchez-Hernandez A, Cortez J, Bazdresch M, Parra-Michel R (2007) Linear Dispersion Codes Generation from Hybrid STBC-VBLAST Architectures. In: Proceedings of 4th International Conference Electrical and Electronics Engineering (ICEEE 2007) 142-145

18. Sandeep G, Ravi-Teja C, Kalyana-Krishnan G, Reddy VU (2007) Low Complexity Decoders for Combined Space Time Block Coding and V-BLAST. In: Proceedings of IEEE Wireless Communications and Networking Conference (WCNC 2007) 582-587

19. Wai WK, Tsui CY, Cheng RS (2000) A Low Complexity Architecture of the V-BLAST System. Proc IEEE WCNC (WCNC 2000) 1:310-314

20. Karray F, Alemzadeh M, Saleh JA, Arab MN (2008) Human-Computer Interaction: Overview on State of the Art. Int J Smart Sensing Intelligent Syst 1(1):137-159

21. Verdú S (1998) Multiuser Detection. Cambridge University Press, The Pitt Building, Trumpington Street, Cambridge, CB2 1RP United Kingdom

22. LiuLiu, Wang Y (2008) Spatially Selective STBC-VBLAST in MIMO Communication System. In: Proceedings of International Conference on Communications, Circuits and Systems (ICCCAS 2008) 195-199, 25-27 May 2008

23. Karniadakis GE, Kirby RM II (2003) Parallel Scientific Computing in C++ and MPI. Cambridge University Press, The Pitt Building, Trumpington Street, Cambridge, CB2 1RP United Kingdom

24. Sandhu S, Paulraj A (2000) Space-Time Block Codes: A Capacity Perspective. IEEE Commun Lett 4(12):384-386

25. Papadias CB, Foschini GJ (2002) On the Capacity of Certain Space-Time Coding Schemes. EURASIP J Appl Sig Process 2002(1):447-458

26. Gorokhov A, Gore DA, Paulraj AJ (2003) Receive Antenna Selection for MIMO Spatial Multiplexing: Theory and Mechanisms. IEEE Trans Signal Process 51(11):2796-2807

27. Ziemer RE, Tranter WH (2002) Principles of Communication: Systems, Modulation and Noise, 5th Edition. John Wiley \& Sons, New York, United States of America

28. Tse D, Viswanath P (2005) Fundamentals of Wireless Communication. Cambridge University Press, New York, United States of America

29. Paulraj A, Nabar R, Gore D (2003) Introduction to Space-Time Wireless Communications. Cambridge University Press, The Pitt Building, Trumpington Street, Cambridge, CB2 1RP United Kingdom

30. Lu J, Letaief KB, Chuang JCI, Liou ML (1999) M-PSK and M-QAM BER Computation Using Signal-Space Concepts. IEEE Trans Commun 47(2):181-184

31. Proakis JG (2001) Digital Communications, 4th edn. McGraw-Hill, New York, United States of America

32. Yang L (2008) Outage Performance of OSTBC in Double Scattering MIMO Channels. Wirel Pers Commun 45(2):225-230

33. Kiessling M, Speidel J (2003) Analytical Performance of MIMO Zero-Forcing Receivers in Correlated Rayleigh Fading Environments. In: Proceedings of 4th IEEE Workshop on Signal Processing Advances in Wireless Communications (SPAWC 2003) 383-387, 2003 